\title{
Advanced optical microscopy methods for in vivo imaging of sub-cellular structures in thick biological tissues
}

\author{
Nanguang Chen ${ }^{* \dagger}$, Shakil Rehman ${ }^{*, \dagger}$ and Colin J. R. Sheppard ${ }^{\ddagger}$, \\ *Department of Biomedical Engineering \\ National University of Singapore, Singapore 117576 \\ ${ }^{\dagger}$ Singapore-MIT Alliance for Research and \\ Technology (SMART) Centre, Singapore 138602 \\ \$epartment of Nanophysics, Istituto Italiano di Tecnologia \\ via Morego 30, 16163 Genova, Italy \\ ${ }^{\S}$ colin@nus.edu.sg
}

Accepted 30 April 2014

Published 4 July 2014

\begin{abstract}
Optical microscopy has become an indispensable tool for visualizing sub-cellular structures and biological processes. However, scattering in biological tissues is a major obstacle that prevents high-resolution images from being obtained from deep regions of tissue. We review common techniques, such as multiphoton microscopy (MPM) and optical coherence microscopy (OCM), for diffraction limited imaging beyond an imaging depth of $0.5 \mathrm{~mm}$. Novel implementations have been emerging in recent years giving higher imaging speed, deeper penetration, and better image quality. Focal modulation microscopy (FMM) is a novel method that combines confocal spatial filtering with focal modulation to reject out-of-focus background. FMM has demonstrated an imaging depth comparable to those of MPM and OCM, near-real-time image acquisition, and the capability for multiple contrast mechanisms.
\end{abstract}

Keywords: Confocal microscopy; multiphoton microscopy (MPM); optical gating; optical coherence tomography (OCT); optical coherence microscopy (OCM); focal modulation microscopy (FMM).

\section{Introduction}

Optical imaging into biological tissue is limited by absorption, and more importantly, by scattering. ${ }^{1}$ Both these processes deplete the light illuminating the specimen, or detected from it. Scattering also diffuses light, so that a good focus or image cannot be formed. Basically, there are two major strategies to overcome the problem. The first, which is the subject of the present review, is to reject the scattered light, by some gating method. In this case, resolution can be in the wavelength range, while the penetration can be a few millimeters. In the second strategy, the scattered light is used to reconstruct

This is an Open Access article published by World Scientific Publishing Company. It is distributed under the terms of the Creative Commons Attribution 3.0 (CC-BY) License. Further distribution of this work is permitted, provided the original work is properly cited. 
an image, methods including diffuse optical tomography (DOT), and in vivo fluorescence imaging. In such cases, penetration can be as large as several centimeters, but resolution is severely limited to the millimeter regime, or worse. As this review is concerned with sub-cellular imaging, we do not discuss it further.

The strengths of absorption and scattering both depend on the wavelength. Absorption is specified by the absorption coefficient $\mu_{s}$, defined as the probability of a photon being absorbed in a unit distance. Blood absorbs strongly for wavelengths below about $600 \mathrm{~nm}$. Absorption of melanin decreases progressively with increasing wavelength. Absorption of water exhibits several windows of comparatively low absorption, at about 480, 805, 1060 and $1250 \mathrm{~nm}$, and can vary by several orders of magnitude in between. In general, it increases as we progress from the visible to the infrared. Scattering is specified by the scattering coefficient, which decreases with increasing wavelength. Overall, for breast tissue, penetration is high in the range $620-1310 \mathrm{~nm}$, and scattering is strong compared with absorption. At shorter wavelengths blood absorption dominates, and at longer wavelengths water absorption dominates. Typical values for $\mu_{s}$ are in the range $0.01-0.1 \mu_{s}^{-1} .^{1}$

Several different gating mechanisms can be used to eliminate scattered light. These are time gating, coherence gating, confocal sectioning, correlation gating, multiphoton sectioning, angular gating, and polarization gating. These approaches are introduced in the following sub-sections.

\subsection{Time gating}

Time gating is the basis of time-domain reflectometry. ${ }^{2,3}$ For biomedical applications, time gating is usually used in a trans-illumination mode. Highly scattered photons travel a greater distance and hence can be identified and removed. This is achieved using an ultrashort-pulsed laser and an ultra-fast time gate, such as a streak camera. ${ }^{4-8}$ The time resolution available is usually not sufficient for microscopic imaging.

\subsection{Coherence gating}

Another way of effectively performing time gating is to make use of the temporal coherence of light. If an interferometer is constructed using broadband light, then interference occurs only when the optical path in the two arms is equal, within the coherence length. In this way, photons that have traveled a further distance can be eliminated, thus resulting in rejection of scattered light, and giving an optical sectioning property that can be used to generate three-dimensional (3D) images. The basic principle of coherence ranging dates back to early 1980 's. ${ }^{9,10}$ An early example of the application to microscopy was given by Davidson et al., ${ }^{11}$ who developed a system based on a Linnik interferometer using white light that they called the coherence probe microscope. Their system was developed for semiconductor device imaging, but is similar to later instruments called full-field optical coherence tomography (FF-OCT) systems. ${ }^{12}$ Optical coherence tomography (OCT) is basically a scanned version of low coherence interferometry. ${ }^{13}$ Optical coherence microscopy (OCM) is also an interferometric imaging method using a broadband light source that uses temporal coherence gating and confocal gating together, to achieve high resolution in thick tissue imaging.

\subsection{Confocal sectioning}

Confocal microscopy (CM) is a scanning microscopy technique in which a pinhole is placed before the detector (see Fig. 1(a)). The pinhole obstructs light that is not focused on a small spot. Such light can come from regions of the sample distant from the focal point, thus resulting in optical sectioning, and in principle improving spatial resolution. Scattered light is also rejected by the pinhole, giving rise to confocal gating, and facilitating imaging in a scattering medium such as tissue. So sectioning and gating are closely related properties. The strength of the sectioning is strongly dependent on the angular aperture of the microscope objective, with a $4 n \sin ^{2}(\alpha / 2)$ variation, where $n$ is refractive index of the immersion medium and $\alpha$ is the angular semiaperture of the lens. ${ }^{14,15} \mathrm{CM}$ can be performed in an epi-illumination, reflection (sometimes called reflectance) geometry, but also in a confocal fluorescence mode. ${ }^{16}$ Confocal fluorescence can rely on chemically or biologically specific fluorescent labels, or intrinsic autofluorescence. Confocal reflection microscopy is widely used for imaging surface structure in industrial applications, but is also used in clinical applications, in ophthalmology and dermatology, for example. It can also be used for 


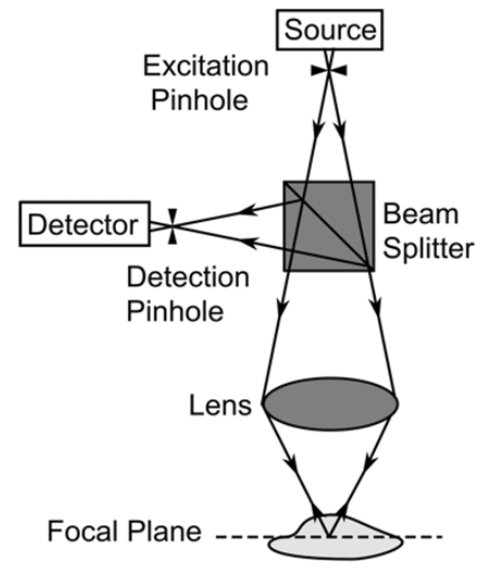

(a)

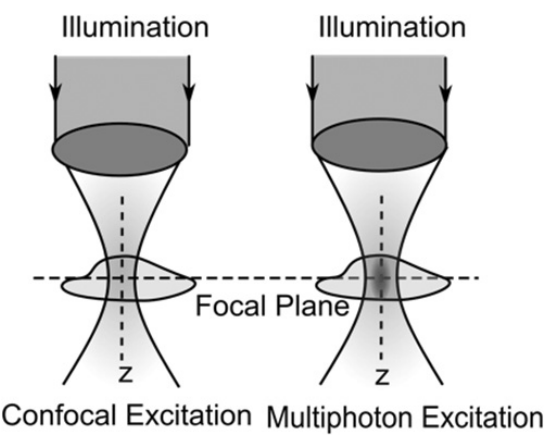

(b)

Fig. 1. The optical setup of (a) confocal system and (b) optical sectioning in confocal and multiphoton excitation.

biological applications using metallic nanoparticles as contrast agents. ${ }^{17,18}$

The sectioning strength of CM can be investigated theoretically using the concept of integrated intensity, the variation with defocus in the intensity integrated over a transverse plane of the image of a point object. ${ }^{19}$ For a conventional microscope, by conservation of energy this must be constant, but for a confocal microscope some energy is intercepted by the pinhole, so that conservation of energy no longer applies. The integrated intensity falls off monotonically, and tends to fall off as $1 / \Delta z^{2}$ for large $z$. For a weakly scattering medium, the diffuse scattering from a layer at a particular depth is proportional to the integrated intensity. So if the illumination intensity is assumed not to decay with depth, the intensity can be integrated in depth to determine the total scattered signal. Schmitt called this the single-backscatter model, and developed it to include depletion of the illuminating beam. ${ }^{20} \mathrm{He}$ found that it gives reasonable predictions to depths of about two scattering lengths, while if depletion is neglected it is reasonably accurate to one scattering length.

It is usually accepted that the confocal microscope was invented by Minsky. ${ }^{21}$ However, the confocal principle seems to have been appreciated earlier, in the work on microphotometry by Koanna and Naora. ${ }^{22,23}$ The image formation process in the confocal microscope is fundamentally different from that in scanning systems without a pinhole. ${ }^{24}$ In some cases the confocal pinhole is replaced by a slit, which allows imaging of a complete line simultaneously, thus increasing imaging speed, but with degraded optical sectioning. ${ }^{25,26}$ In fact, a confocallike system of this type was reported in $1940 .{ }^{27}$ Another way to increase scanning speed is to use an array of pinholes, as in the tandem scanning (or spinning disk) microscope. ${ }^{28,29}$ This also degrades the optical sectioning because of cross-talk between the pinholes. ${ }^{25,30}$ For a confocal system with a pinhole, the defocus signal decays as $1 / \Delta z^{2}$, whereas for a slit system it decays as $1 / \Delta z$ and for a spinning disk system it tends to be a constant value, the value of which depends on the aperture spacing. For a pinhole system, the sectioning, and therefore also the gating strength, is weaker for a larger pinhole, ${ }^{31-34}$ but too small pinhole results in weak signal and strong coherent artifacts in a scattering medium. ${ }^{20,35-37}$ It should be noted that it is not the absolute size of the pinhole that is significant, but its size relative to that of the Airy disk formed in the pinhole plane by the focused wave.

The confocal pinhole can also be replaced by a single mode optical fiber. ${ }^{38-40}$ The size of the fiber core and the geometry now determine the sectioning strength. ${ }^{41,42}$ Confocal sectioning arises because the sample is illuminated with a focused spot. This is a special case of structured illumination, which includes other examples such as full-field illumination with a pattern of fringes. ${ }^{43,44}$ Structured illumination has been shown to give superior $3 \mathrm{D}$ resolution compared with $\mathrm{CM},{ }^{45,46}$ but has been found to not penetrate so well into scattering media.

\subsection{Correlation gating}

Optical gating with performance similar to confocal sectioning may results from a correlation effect (Fig. 2). If a wave interferes with a reference beam on a detector, the interference signal selects the signal wave front that matches the reference beam 
wave front. ${ }^{47}$ By using a spherically expanding reference beam, light coming from a point in the sample can be detected, without a lens being necessary; ${ }^{48}$ or by using a lens and a focused reference beam a 'synthetic' confocal pinhole can be generated. ${ }^{49,50}$ By combining with broad-band illumination, both confocal and coherence gating result. ${ }^{36,51}$

This correlation effect of interference can also be used in a full-field microscope (Fig. 2(a)). The coherence probe microscope of Davidson et al. ${ }^{11}$ thus also exhibits sectioning from the correlation effect, in addition to the coherence sectioning.

In a similar way, OCT when performed with a high numerical aperture (NA) lens, gives both coherence and confocal gating. This is then called OCM. ${ }^{52}$ A major difference between OCM and OCT

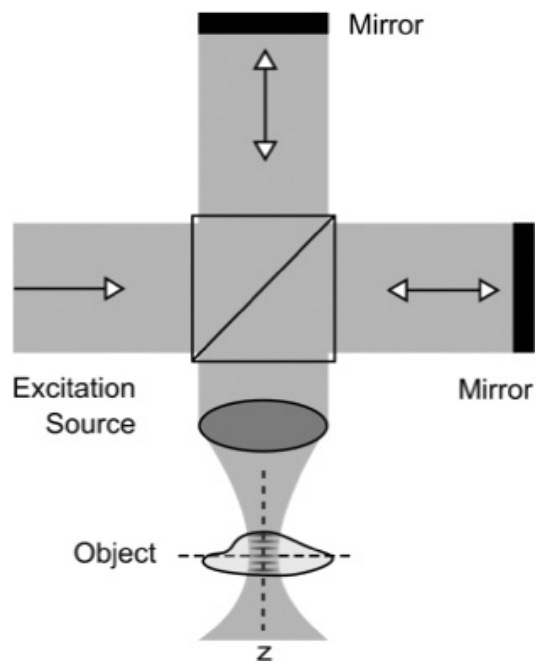

(a)

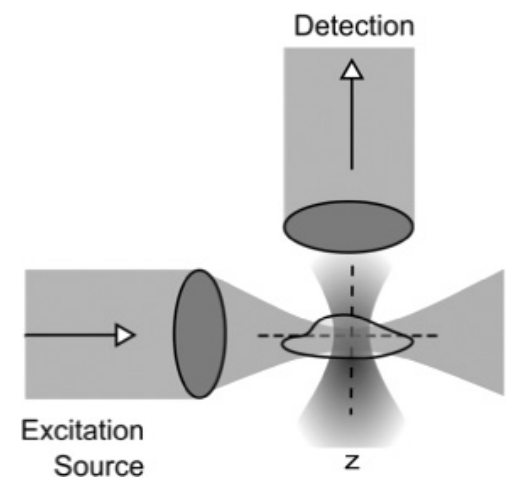

(b)

Fig. 2. Optical sectioning through interferometric scheme (a) is used in correlation gating and coherence gating (when using a broadband source) and (b) angular gating as explained in Sec. 1.5. is that in OCM it is necessary to scan in $3 \mathrm{D}$, whereas in OCT the low coherence provides sectioning within the depth of focus of the imaging lens.

\subsection{Angular gating}

Confocal imaging is basically a geometric effect, related to the angular aperture of the illumination and collection. But it is also possible to use angular gating in the Fourier domain. This general approach has a long history. Siedentopf and Zsigmondy proposed the ultra-microscope, in which illumination is in a direction transverse to the optical axis of the imaging system. ${ }^{53}$ This is like a form of dark field microscope, allowing weak scattering from sub-wavelength particles to be observed. Goldman used a development of this concept in his confocal slit system. ${ }^{27}$ The illumination and imaging axes are tilted with respect to one-another, with the result that a particle must be in both the illumination and collection paths in order for scattering to be detected. This has the effect of increasing the overall optical sectioning. In a similar way, for a scattered photon to be detected, it must diffuse from the illuminated region to the collection cone, and at the same time be oriented in the correct direction. This principle has been widely used in the ophthalmology specialization, in the so-called slit lamp. Maurice divided the objective aperture into two semicircular halves, one for illumination, and the other for collection. ${ }^{54} \mathrm{He}$ also used this principle in his form of the scanning slit microscope. ${ }^{55}$ The divided aperture method was further refined by Koester, ${ }^{56,57}$ and more recently by Dwyer et al. ${ }^{58,59}$ Analysis of the imaging performance shows that the sectioning strength can be improved, so that the defocus signal falls off as $1 / \Delta z^{3}{ }^{60-62}$ The divided aperture method can also be used with fluorescence microscopy. ${ }^{63}$ A similar off-axis instrument using two separate objectives, called the theta microscope, has also been used with confocal fluorescence microscopy,${ }^{64}$ or for reflection imaging, sometimes under the name of the dual-axis microscope. ${ }^{65-69}$ Other geometries can also be used. For example, using annular apertures in conjunction with a confocal pinhole of finite size can also result in improved sectioning, ${ }^{35,70-73}$ and this approach can also be used with confocal fluorescence microscopy. ${ }^{74,75}$

A rapidly developing approach is based on the illumination by a plane of light. These techniques are variously called light sheet microscopy, 
orthogonal-plane fluorescence optical sectioning (OPFOS),${ }^{76}$ selected plane illumination microscopy (SPIM), ${ }^{77-79}$ or just ultra-microscopy ${ }^{80}$ (named after the original paper by Siedentopf and Zsigmondy ${ }^{75}$ ). The light sheet can be generated using a scanned beam, and combined with the structured illumination technique. ${ }^{81}$ A scanned Bessel beam has also been used, usually combined with multiphoton excitation to reduce the side lobe level. ${ }^{82-84}$ Light sheet microscopy has also been combined with confocal gating. ${ }^{85}$

The performance of angular gating combined with confocal gating has been explored, and justified, using Monte-Carlo methods. ${ }^{37,69,73,86,87}$

\subsection{Polarization gating}

Light tends to lose its well-defined polarization upon multiple scattering. So imaging between crossed polarizers can be used to reject scattered light. As the scattered light is unpolarized, the intensities of the parallel-polarized and perpendicular-polarized components are equal, and therefore an efficient gating method is to subtract the intensity of the perpendicular-polarized component from that of the parallel-polarized component, to give the pure polarized component. ${ }^{110}$ Combinations of polarization gating with time-gating, ${ }^{111}$ and angular gating have been reported. ${ }^{8-90,95,112}$

\section{Multiphoton Microscopy \\ 2.1. Optical sectioning by MPM}

MPM relies on a nonlinear interaction within the sample. If more than one photon is involved in the process, the illumination point spread function is sharpened up, thus introducing an optical sectioning effect, as shown in Fig. 1(b). The general principle of exploiting the high power in a focused laser spot to construct a scanning microscope based on a nonlinear interaction was proposed by Sheppard and Kompfner. ${ }^{91}$ Specific examples of nonlinear processes that could be used were mentioned, including harmonic generation, frequency mixing, two-photon fluorescence and coherent Raman scattering. It was also described how for a fixed average input power, the signal can be increased by concentrating the illumination in time as well as space, by using pulsed-beam illumination. Imaging by second harmonic generation (SHG) using a full-field microscope, rather than a scanning system, was demonstrated by Hellwarth and Christiansen. ${ }^{92,93}$ In this case the emitted photons are imaged, and no optical sectioning results. But SHG in a scanning system does exhibit optical sectioning, as the focused illumination intensity is effectively squared by the two-photon process. ${ }^{94,95}$ SHG microscopy was first applied to biological imaging by Freund et al. ${ }^{96,97}$ Coherent anti-stokes Raman spectroscopy (CARS) microscopy was first demonstrated by Duncan et al. ${ }^{98}$ Two-photon fluorescence microscopy was demonstrated by Denk et al. ${ }^{99}$ and they filed a patent for two-photon fluorescence microscopy using pulses shorter than $1 \mathrm{ps}^{100}$ Other mechanisms that have been used for MPM include third harmonic generation $^{101}$ and three-photon fluorescence. ${ }^{102-104}$ Different modes of MPM can also be combined. ${ }^{105}$

MPM has several advantages over CM. It usually uses excitation in the near infrared, which penetrates into tissue better than the visible light used for single-photon fluorescence, although resolution for the same absorption transition energy is inferior to the single-photon case. ${ }^{18,106}$ Excitation by scattered light is much weaker than in singlephoton microscopy because of the power law dependence, so penetration is deeper. But when the illumination spot is depleted by extinction, the strength of the multiphoton interaction decreases more rapidly than it does in the single-photon case. $^{107}$ The fluorescent light has only to be collected, rather than imaged as in conventional or confocal fluorescence microscopy, so scattering of the fluorescent light is not so important. Another advantage of multiphoton fluorescence is that photobleaching, a serious consideration in singlephoton fluorescence, is much reduced because multi-photon absorption mainly occurs in the focal plane. Harmonic generation, on the other hand, does not suffer from fading.

Multiphoton signals are often weak, so collection efficiency is often improved using a nondescanned detector. MPM can be combined with a confocal pinhole, ${ }^{108-112}$ to improve the overall optical sectioning strength, but then use of a nondescanned detector is not possible.

Nonlinear contrast is based on second- and thirdorder (or higher) nonlinear light-matter interactions that, with a high NA microscope objective, are possible only at the focus. These nonlinear optical effects are proportional to the second or third power of the illumination light intensity, and therefore the 
interaction of light with a sample takes place only at the focal plane, rejecting out-of-focus light and resulting in an optical sectioning property that is unique to nonlinear excitation. The principle of nonlinear fluorescence microscopy is based on simultaneous absorption of two or more photons, as in two-photon excited fluorescence microscopy ${ }^{99}$ and three-photon excitation fluorescence microscopy. ${ }^{102,113,114}$ When the phase of the generated signal in a nonlinear microscope is determined by the phase of the excitation field and the geometrical distribution of radiating molecules, the process is coherent. Some of the coherent nonlinear microscopic techniques are SHG microscopy, ${ }^{115-118}$ CARS microscopy ${ }^{119}$ and third harmonic generation (THG) microscopy. ${ }^{63,120-123}$ MPM is based on the efficient generation of ultrashort pulses. Advances in the production of ultrashort-pulsed lasers have significantly contributed to the development of these nonlinear optical modalities.

Deep tissue imaging in thick samples using MPM depends on factors such as laser power, pulse repetition rate, pulse width and wavelength. The imaging depth in multiphoton excitation is proportional to the absorption coefficient of the light in a sample, and changes logarithmically with average power, collection efficiency and duty cycle. Multiphoton fluorescence imaging has been reported up to $1 \mathrm{~mm}$ using a regenerative amplifier with $200 \mathrm{~nJ}$ pulse energy at a repetition rate of $200 \mathrm{kHz} .{ }^{124} \mathrm{By}$ reducing the pulse width, increased two-photon fluorescence excitation at greater depths can be achieved with dispersion compensation of laser pulses. ${ }^{125}$ But as the laser pulse width is reduced, the width of the laser spectrum increases, thereby exceeding the bandwidth of the two-photon excitation spectrum of the dye, and thus limiting the benefits of shorter pulses. ${ }^{126}$

Longer wavelength can be used to achieve deeper penetration depth in MPM. Multimodal imaging at $1230 \mathrm{~nm}$ excitation wavelength has been used to image plant cells and zebra fish embryos. ${ }^{127,128}$ Improved imaging depth has also been reported using $1300 \mathrm{~nm}$ excitation, as compared to $800 \mathrm{~nm}$ excitation wavelength, due to lower scattering in tissue, with two-photon fluorescence microscopy, ${ }^{129}$ and (in skin tissue) for SHG microscopy. ${ }^{130} \mathrm{Up}$ to $1.6 \mathrm{~mm}$ imaging depth has been reported in in vivo imaging of adult mouse brain at $1280 \mathrm{~nm}$ using 2-photon fluorescence, ${ }^{131,132}$ and to $1.3 \mathrm{~mm}$ at $1675 \mathrm{~nm}$ using 3 -photon fluorescence. ${ }^{133}$

\subsection{Multifocal multiphoton microscopy}

MMM is a parallelized multiphoton imaging method for real-time nonlinear imaging applications in which, a beam of ultrafast femtosecond pulses is split into several lower energy ones, generating simultaneously multiple and well-separated foci. MMM uses more of the available laser power and at the same time it parallelizes the imaging process without significant loss of resolution. ${ }^{134-136}$

For biomedical applications high-speed MPM can be achieved by incorporating high-speed scanners such as polygonal mirror scanners or resonant mirror scanners ${ }^{137,138}$ instead of galvanometric mirror scanners used in conventional multiphoton microscopes. These approaches can achieve a scanning speed of up to 30 frames per second in thick tissues but are limited by the signal-to-noise ratio (SNR). Another method to improve speed is to scan selected regions of interest in the sample rather than the whole volume. Using acousto-optic deflectors for fast point scanning, however, has provided limited improvement due to significant dispersion of ultrashort pulses and spherical aberrations introduced by the optical elements used. ${ }^{139}$

In MMM, the sample is scanned with multiple excitation focus points, ${ }^{134-136}$ or lines ${ }^{140}$ rather than a single excitation focus. The emitted light is collected by the multiple foci on a charge-coupled device (CCD) camera. The CCD integrates the collected photons from the sample in a $2 \mathrm{D}$ scan. In this method, the number of excitation foci determines the imaging speed of MMM, leading to a high frame rate without increasing the excitation power, thereby reducing photo-toxicity. To obtain deep tissue imaging, higher laser power is required to compensate the signal loss in tissue. Imaging depth in thick tissues can be improved by a method based on multi-anode photomultiplier tubes. ${ }^{141,142}$

\subsection{Temporal focusing}

Temporal focusing of femtosecond laser pulses is used to reduce the background excitation in MPM and for improving the penetration depth of optical imaging in highly scattering biological tissue. Understanding the spatio-temporal focusing of ultrashort pulses through highly scattering media is critical in MPM applications. Most of the applications of ultrashort pulses need focusing through lenses, and a transform-limited temporal profile, 
along with a diffraction-limited focused spot size, is required. It is well known that complex spatiotemporal distortions can occur at the focus of a lens due to spherical and chromatic aberrations. ${ }^{143}$ Control and manipulation of ultrashort pulses when aberrations are present is needed for effective use of the pulses in a variety of applications such as multiphoton imaging, photonics and communications.

Temporal focusing of ultrashort pulses can be applied in the wide-field depth-resolved two-photon fluorescence microscopy, where a plane in the sample is excited selectively by using geometrical dispersion of an ultrashort pulse. The effects of spatial modulation of a temporally focused excitation pattern on both the generated excitation pattern, and on its axial confinement, have been studied. ${ }^{144}$ A geometric optics model with MonteCarlo scattering simulations have been applied to study the temporal focusing characteristics in scattering and nonscattering media. The concept of simultaneous spatial and temporal focusing of femtosecond pulses ${ }^{145,146}$ has been used to reduce the background excitation in MPM for imaging in highly scattering biological specimens. ${ }^{147}$ Spatially and temporally resolved measurement of a speckle field produced by the propagation of a ultrafast optical pulse through a thick, strongly scattering, medium can be used for imaging in MPM applications. ${ }^{148}$

\subsection{Other nonlinear microscopy modalities}

Some of the nonlinear optical microscopic techniques are described below.

\subsubsection{SHG microscopy}

SHG was first applied in optical microscopy to image the structure of nonlinearities in a crystal of ZnSe in a full field illumination setup ${ }^{149}$ and later the scanning mode of a SHG microscope was also demonstrated. ${ }^{117}$ The first biological application of SHG microscopy was the imaging of rat-tail tendon. ${ }^{96}$ The development of stable, high-power, femtosecond mode-locked lasers has contributed to the efficient generation of nonlinear optical responses. Now SHG microscopy is used in a variety of biological applications. Optical microscopy with SHG has been widely used for imaging collagen, ${ }^{150-153}$ myocytes ${ }^{152,154}$ and plants. ${ }^{155-157}$
SHG is a resonant scattering process as shown in Fig. 3(d). SHG microscopy is based on noncentrosymmetric organization of microstructures in the sample, and SHG signal can be generated due to a broken symmetry at an interface or due to a noncentrosymmetric arrangement within bulk structures. At an interface, biological membranes can produce detectable SHG signal as in the case of a lipid bilayer. ${ }^{158}$ Molecular structures having a symmetric distribution of chirality in the membrane do not give rise to SHG signal: only an ordered asymmetric distribution of chiral molecules in the membranes is responsible for SHG. ${ }^{115} \mathrm{SHG}$ can also be produced in biogenic crystal structures, for example, in calcite or starch granules, ${ }^{155}$ and bio-photonic crystalline and semi-crystalline structures in living cells. ${ }^{159}$

\subsubsection{THG microscopy}

Third harmonic light can be generated at an interface by a tightly focused laser beam. It is possible to image both biological and nonbiological specimens, with the inherent optical sectioning property of THG microscopy. Even though, unlike SHG, THG can occur in symmetric crystal structures, with an isotropic structure the harmonic generated on either side of focus cancels out. Third harmonic signal in a material is produced only when the axial symmetry at the focal plane can be broken by a change in the material properties, such as interfaces and boundaries due to refractive index or nonlinear susceptibility changes. The localized production of third harmonic generation signal at material interfaces provides the inherent optical sectioning desired in $3 \mathrm{D}$ imaging at higher axial resolution. It is possible to produce 3D images by THG from different planes perpendicular to the axis of beam propagation. Compared with the other modes of single or multiphoton fluorescence microscopy (Fig. 3(a-c)), external labeling or fluorophores are not required in THG microscopy. THG is also a resonant energy transfer process (Fig. 3(e)), like SHG and CARS.

Under tight focusing conditions, THG can be generated through an interface within the focal volume of the excitation beam. ${ }^{160}$ It has been shown that whenever there is either a change in refractive index or third-order nonlinear susceptibility, third harmonic light is produced and as a result of this interface effect third harmonic imaging is possible and can be applied to study transparent samples. ${ }^{101,120}$ Volumetric imaging has also been 


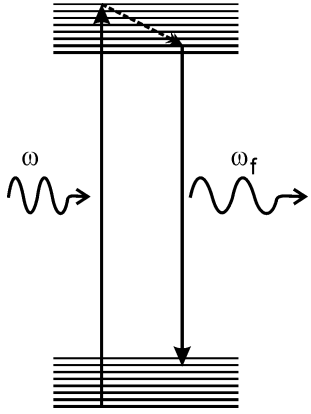

(a)

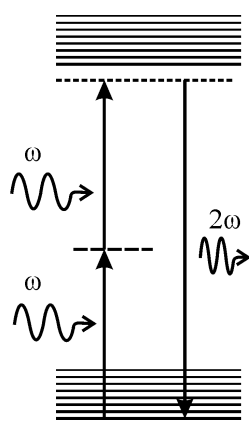

(d)

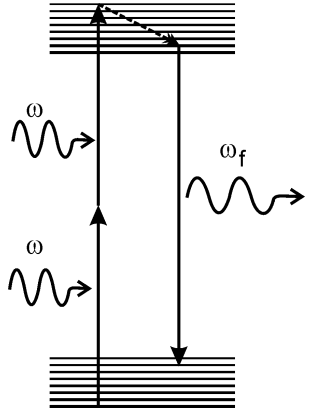

(b)

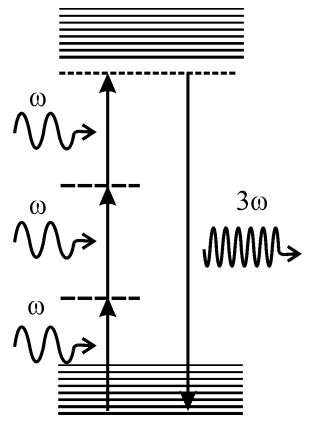

(e)



(c)

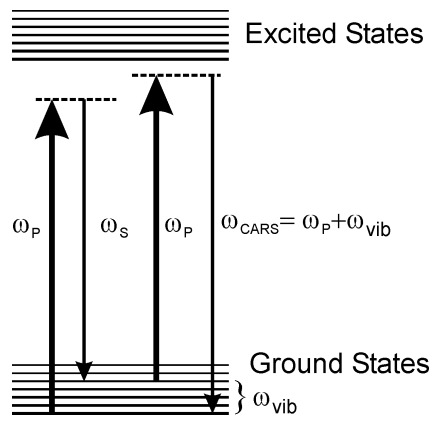

(f)

Fig. 3. Fluorescence emission by: (a) single-photon excitation (b) two-photon excitation and three-photon excitation. Fluorescence is characterized by nonresonant processes resulting in incoherent emission. The coherent scattering processes are shown by resonant emission from (d) two-photon absorption as in SHG, (e) three photon-absorption as in THG and (f) CARS.

performed in both biological and non-biological specimens, demonstrating the dynamical imaging properties of THG in live samples. ${ }^{121,161,162}$ Third harmonic generation microscopy is shown to be particularly suitable for imaging biogenic crystals and polarization sensitive imaging of crystalline structure in biological samples. ${ }^{159,163}$ THG has been used for the characterization of saline solutions and structural changes in collagen. ${ }^{164}$ It is shown that THG epidetection is generally possible when the sample structure is embedded in a scattering, nonabsorbing tissue with thickness greater than the reduced scattering mean free path. ${ }^{165}$ Multimodal microscopic techniques have also been reported by a combination of THG, SHG and two-photon fluorescence contrast methods on the same microscope. ${ }^{166}$

\subsubsection{Coherent anti-stokes Raman scattering}

CARS microscopy is based on the use of two different laser beams, the pump and Stokes beams, that are tuned to match the energy gap between two vibrational levels in a molecule as shown in Fig. 3(f).
CARS is used to probe chemical bond vibration levels and provides molecular specificity, particularly for small molecules. ${ }^{119,167}$ Stronger vibrational signals can be obtained with CARS microscopy, that is a nonlinear Raman scattering method, as compared with conventional Raman scattering microscopy. CARS is a four-wave mixing process, in which a pump beam at frequency $\omega_{P}$ and a Stokes beam at frequency $\omega_{S}$ interact with the sample to generate an anti-Stokes signal at frequency $\omega_{\mathrm{AS}}=2 \omega_{P}-\omega_{S}$. This expression can also be written as $\omega_{\mathrm{AS}}=\omega_{P}+$ $\omega_{\text {vib }}$, when the beat frequency between the pump and Stokes beams, $\omega_{P}-\omega_{S}$, matches the frequency of a particular Raman active molecular vibration, $\omega_{\text {vib }}$. This results in an enhanced anti-Stokes signal, providing the vibrational contrast for CARS microscopy. ${ }^{168}$ The anti-Stokes Raman signal $\omega_{\mathrm{AS}}$ is represented as $\omega_{\text {CARS }}$ in Fig. $3(\mathrm{f})$. CARS can selectively image molecules by exciting their specific vibrational modes with high sensitivity. Since its signal frequency is blue-shifted from the excitation frequencies, CARS can be used in the presence of high fluorescence background. Normally the pump and Stokes fields are generated by a near infrared 
laser, which can penetrate deeply into biological system with little absorption. This permits CARS to be noninvasive in imaging living cells.

Raman scattering is a unique method for live cell imaging, based on chemical contrast without using fluorescent markers. Inherent Raman signals do not photo-bleach and allow long-term cellular studies. Developments in CARS microscopy have offered further improvement in imaging, such as (a) F-CARS, forward-detection with parallel-polarized pump and Stokes beams, (b) E-CARS, epi-detection with parallel-polarized pump and Stokes beam, (c) P-CARS, forward-detection with polarization, and (d) C-CARS, detection with counter propagating parallel-polarized pump and Stokes beams. These four kinds of the CARS methods can be applied under different circumstances. F-CARS microscopy produces a large signal at low excitation power. It is suitable for a vibration with a large resonant CARS signal. However, the resonant F-CARS signal is often overwhelmed by the nonresonant background from scatterers and solvent. The E-CARS and C-CARS beam geometries introduce a phase mismatch, which acts as a size filter that effectively rejects the signal from the bulk solvent. E-CARS permits high-sensitivity imaging of small features embedded in a nonlinear medium.

These nonlinear microscopic modalities have different properties for imaging thick biological tissue. SHG, THG, CARS, two- and three-photon fluorescence give deeper penetration but are limited by the wavelength and dispersion of ultrashort pulse propagation through thick biological tissue. OCT, OCM and FMM are new techniques for in vivo imaging of thick biological tissue at greater depths than the nonlinear methods and are being developed for potential applications in biomedical field.

\section{Optical Coherence Microscopy}

Optical imaging in scattering media such as biological tissue is a fundamentally difficult problem. Low coherence light and ultrashort laser pulses can be used to image thick biological tissue at greater depths. Time-of-flight information of a light signal scattered from a tissue contains spatial information about the fine structure of the specimen. Optical ranging with a low coherence light source can be used in microscope modalities such as OCT and OCM. ${ }^{13,169,170}$ The axial resolution of OCT is determined by the coherence length of the light source, and the lateral resolution is controlled by the NA of the imaging optics. The combination of OCT with high NA optics gives a combined depth sectioning by utilizing the coherence gating and the confocal gating, and is called OCM.

OCM is an interferometric imaging method that works on the basic principle of splitting and recombining the optical beams of a broadband light source. The interferometric setup allows rejecting scattered photons on the basis that they do not contribute to the fringe pattern. The OCM optical scheme is similar to that of low coherence interferometry, that is used in thickness and refractive index measurements. ${ }^{171,172}$ A typical OCM setup for imaging thick biological tissue based on a Michelson interferometer configuration is shown in Fig. 4. Light from a low coherence source such as a super-luminescent diode (SLD) is split in two paths, one traverses the reference arm and reflects from a reference mirror, while the other part of the light illuminates the sample via a microscope objective. Due to the broadband of the light source, interference between the light from both arms is observed only when their optical path lengths are matched to within the coherence length of the source. The temporal coherence length of the light source determines the depth resolution, and scanning the reference arm allows different depths within the sample to be sectioned.

The heterodyne detection and coherence gating used in OCM increases the penetration depth in thick tissue by a factor of two to three, as compared to that of a confocal microscope. Heterodyne detection is the application of a frequency shift between the sample and reference beams during detection. It is commonly used in OCT and OCM to overcome the noise issues in optical detectors. ${ }^{173}$ The most common method of implementing heterodyne detection is through the use of a delay line, which shifts the coherence gate, or using a moving reference mirror. Delay lines are used to change the geometrical length of the light path using stepper motors, loudspeaker cones and other methods. ${ }^{13}$ Piezoelectric fiber stretchers have been used to avoid mechanical vibrations, but with the drawbacks of temperature effects and dispersion. ${ }^{174,175}$ Coherence gate modulation has also been achieved by pulse shaping in the spectral domain, ${ }^{176}$ and phase delay lines. ${ }^{177,178}$

Recently, the similarities and differences between OCT, OCM, FF OCT, confocal interferometry, interferometric synthetic aperture microscopy 


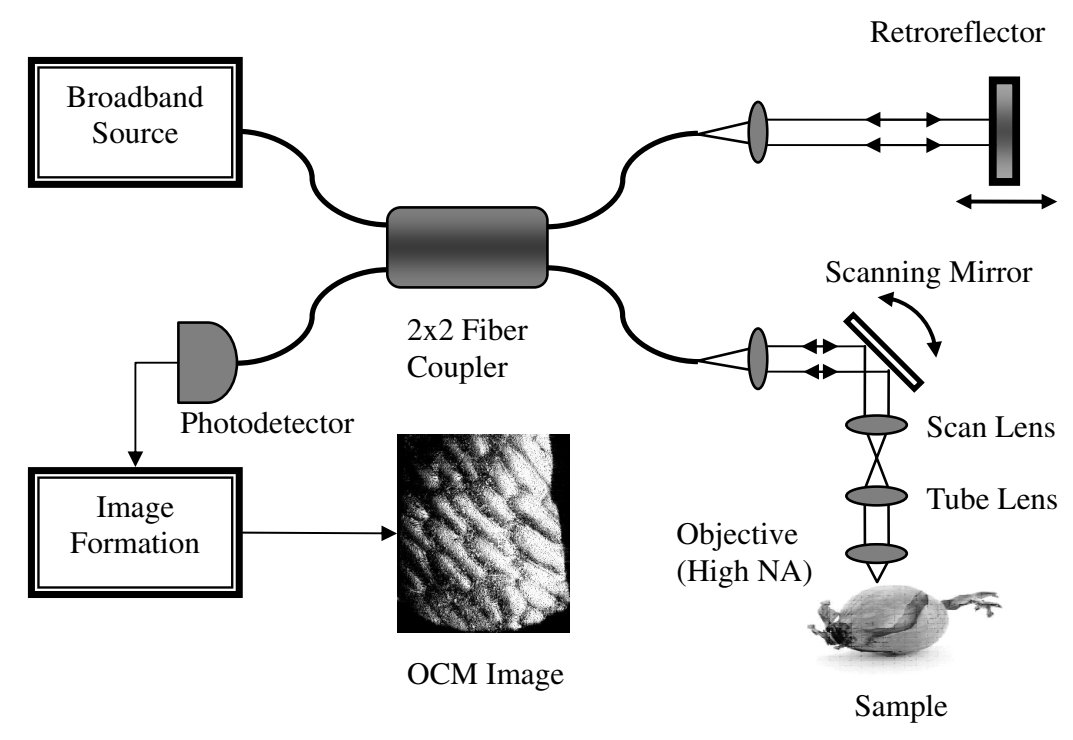

Fig. 4. A typical optical scheme of OCM.

(ISAM), and digital holographic microscopy within the framework of the coherent transfer function have been pointed out. ${ }^{179}$ In OCM, high NA lenses are used, such that the confocal sectioning for each spectral component can be described by a $3 \mathrm{D}$ coherent transfer function that is identical to that of a confocal interference system of the same geometry. In conventional OCT, the apertures of the illuminating and collecting pupils are small and equal. If the aperture is small enough so that we can neglect the contribution to longitudinal resolution from the confocal effect, the longitudinal resolution comes only from temporal coherence effects. In OCT using a single-mode fiber-optic architecture, the fiber mode acts as a synthetic pinhole in the detection process. $^{38,39,180}$

A model for image formation in OCT and OCM in the Fourier domain, using the principle of the generalized aperture and the coherent transfer function has been reported, ${ }^{181,182}$ in which it was proposed that a model-based approach could be used to overcome the limited depth of focus in Fourier domain OCM. Full field optical coherence microscopy (FFOCM) combined with structured illumination fluorescence microscopy has been used to co-localize reflectance and fluorescence images in a fixed and labeled mouse retina. ${ }^{183}$

\subsection{Dynamic focusing}

To achieve high lateral resolution in an OCT setup, a high NA lens is used in order to obtain coherence gating and confocal gating at the same depth position during axial scanning. This method provides what is known as dynamic focusing for high resolution in an OCM.

Dynamic focusing is used in such microscopes to achieve invariant resolution across the entire scan depth. A common translation stage for both the reference and sample arm is used to ensure invariant resolution throughout the lateral and depth scans. ${ }^{184}$ By keeping the arms of the interferometer stationary, and moving the sample laterally at each scan, an axially magnified focused beam can be scanned and compensated for the path length changes caused by the refractive index of the sample. ${ }^{185}$ A dynamic focusing system based on a deformable micro-electro-mechanical systems (MEMS) mirror has been designed in which the MEMS mirror deforms in order to shift the beam focus in the sample in correspondence with the coherence gate of each lateral scan. ${ }^{186}$ Dynamic focusing by using a liquid-filled polymer lens whose curvature can be changed by hydraulic pressure to obtain the variable focus has been proposed. ${ }^{187} \mathrm{~A}$ microscope objective has been designed to minimize aberrations by incorporating a dynamic focusing system within the microscope objective. ${ }^{188}$

\subsection{Dark field OCM}

Dark field imaging is used in wide-field microscopy for achieving contrast based on scattering in the sample. Dark field OCM has been introduced to 
improve the sensitivity of OCM for the imaging of weakly scattering objects. ${ }^{189}$ In this method, the use of separate illumination and detection apertures ensures the suppression of signal created by specular reflections from flat surfaces within the sample.

Based on a coherent optical transfer function analysis, ${ }^{182}$ it has been shown that dark field OCM has a transfer function that shows improved transverse spatial frequency content. ${ }^{181}$ Another advantage of dark field OCM is that the illumination power can be increased to improve the signal level of high spatial frequency content, which is not possible with classical OCM where the illumination power is limited by the signal level of the low spatial frequencies that use most of the available dynamic range of the detection system.

\subsection{Photon noise limitations}

The speed of data acquisition and the SNR in OCM are both limited by fundamental photon noise. The photon shot noise is linked to the quantum nature of light, as most OCT/OCM sources (SLDs, broadband lasers) follow Bose-Einstein statistics, while quasi-monochromatic lasers follow Poisson statistics. These two phenomena tend to approach each other at low photon numbers, and hence the distinction becomes crucial at higher intensities. The low coherence sources such as SLDs usually work at power levels and coherence times that lead to BoseEinstein photon bunching. The photon noise in such low coherence sources is dominated by this photon bunching effect. In OCM one can reduce the effect of photon bunching by attenuating the reference beam power, thereby bringing the instrument close to the regime of Poisson noise. An increase in the sample beam power increases the SNR when imaging biological tissue, but the reference power must not be allowed to increase into the photon-bunching regime. There are several other noise sources arising from the use of electronic circuits in the OCM systems, such as $1 / f$ noise, Johnson noise, dark current, pre-amplifier noise, and analog-to-digital conversion (ADC) noise. ${ }^{190}$

\section{Focal Modulation Microscopy}

Focal Modulation Microscopy (FMM) has been recently developed as a new confocal method for in vivo imaging of thick biological tissues. ${ }^{191,192}$ It is compatible with a range of contrast mechanisms, including single-photon excited fluorescence, twophoton excited fluorescence, and back scattering/ reflection. It has been demonstrated theoretically and experimentally that FMM can achieve an imaging depth significantly greater than CM, especially when the image quality in deep regions is limited by the strong background that results from multiple scattering.

\subsection{Principle and theory}

FMM essentially provides an effective background rejection mechanism based on the coherence properties of the excitation light beam. It does not, however, rely on the coherence between the excitation beam and the emission beam. The latter makes it possible for FMM to be applied to fluorescence imaging. In this section, we review the basic principle and theoretical background of singlephoton fluorescence-based FMM.

A simplified theoretical model has been developed to investigate the image formation process for both CM and FMM. ${ }^{193}$ Contributions from ballistic photons and scattered photons can be separated and treated with different physical models. The nonscattered photons in the excitation or emission light form the so-called ballistic component. Its propagation can be modeled using the scalar diffraction theory. For example, the intensity distribution of the ballistic illumination light focused by the objective lens, as a function of the spatial location $\mathbf{r}$, is given by

$$
I_{B}\left(\mathbf{r}, \mathbf{r}_{F}\right)=\mathrm{e}^{-\mu_{l} z} C\left|\int_{0}^{1} J_{0}(v \xi) \mathrm{e}^{i u \xi^{2} / 2} \xi d \xi\right|^{2} .
$$

Here $\mathbf{r}_{F}=\left(x_{F}, y_{F}, z_{F}\right)$ is the location of the focal point of the objective lens with its focal length given by $z_{F}$. The normalized optical coordinates $v=k \rho N A$ and $u=4 k n \Delta z \sin ^{2}(\alpha / 2)$, where the NA is $N A=$ $n \sin (\alpha), k=2 \pi / \lambda$ is the wave number, $n$ is the refractive index of the imaging medium and $\alpha$ is the semi-aperture angle of the lens, are related to the radial displacement $\rho=\sqrt{\left(x_{F}-x\right)^{2}-\left(y_{F}-y\right)^{2}}$, and the defocus $\Delta z=z-z_{F}$, respectively. $J_{0}(\cdot)$ denotes the zero-order Bessel function. The constant $C$ is used to normalize the total excitation power to unity. Attenuation of the ballistic component due to scattering and absorption in the medium is modeled by the first exponential term, which depends on the extinction coefficient $\mu_{l}=\mu_{a}+\mu_{s}$ (the sum of 
absorption and scattering coefficients) and the penetration depth $z$. The same wave optics model is applicable to the propagation of the ballistic emission from within the sample to the pinhole detector.

For scattered photons, it is assumed that they lose spatial and temporal coherence and can be treated as classical particles. Monte Carlo simulation is a useful tool to trace migration of scattered photons in the sample. Their propagation outside the sample can be traced using geometric optics.

The ballistic excitation light can be well concentrated within the diffraction limited focal volume. On the other hand, the ballistic emission from the focal volume can be adequately differentiated from out-of-focus light by a confocal pinhole. Among the detected emission photons, those related to ballistic excitation and emission contribute to the desired signal, while the rest lead to a background that adversely affects the image quality in terms of contrast and spatial resolution.

For a conventional CM setup, the desired signal decays rapidly with the penetration depth

$$
S_{\mathrm{CM}} \propto \mathrm{e}^{-2 \mu_{l} z}=\mathrm{e}^{-2 z / l_{t}},
$$

where the typical value for $l_{t}=1 / \mu_{t}$ is around $100 \mu \mathrm{m}$ in biological tissues. The background $B_{\mathrm{CM}}$, however, includes contributions from scattered light and attenuates at a much slower pace when the focal point moves into the medium. As a result, the signal can be overwhelmed by the background ratio when the imaging depth is greater than $200 \mu \mathrm{m}$.

FMM introduces a Spatial-Temporal Phase Modulator (STPM) in the excitation light path. The modulated excitation field at the back aperture of the objective lens is given by

$$
E(\mathbf{r}, t)=\mathrm{e}^{i H(r, t)} E_{0}(\mathbf{r}, t),
$$

where $E_{0}(\mathbf{r}, t)$ usually represents a Gaussian or uniform beam entering the STPM. The modulation function $H_{0}(\mathbf{r}, t)$ is the additional phase shift introduced by the modulator and is a function of time $t$ and the two-dimensional vector $\mathbf{r}=[x, y]$ in the aperture plane. In an example implementation, the aperture is divided into two concentric zones of equal area, and $H(\mathbf{r}, t)$ is given by

$$
H(\mathbf{r}, t)=\left\{\begin{array}{cc}
\phi_{0}, & \mathbf{r} \notin R_{1}, \\
\phi_{0}+\pi \cos \left(f_{0} t\right) / 2 \pi / 2, & \mathbf{r} \notin R_{2}
\end{array},\right.
$$

where $f_{0}$ is the modulation frequency, $R_{1}$ is the inner circular zone where the phase delay is a constant, while $R_{2}$ is the annular zone in which the relative phase delay varies from 0 to $\pi$ periodically.

The spatio-temporally phase modulated excitation beam, when brought to the focal point of the objective lens, generates an intensity modulation around the focal volume. This results in an oscillatory component in the fluorescence emission as well. When the focal point is located within a turbid medium, however, only the ballistic excitation light contributes to the modulated fluorescence excitation and emission. Scattered excitation photons are considered incoherent and they lead to a constant background. This is the key idea behind FMM for background rejection. In the pinhole detector output, only the $\mathrm{AC}$ component at the modulation frequency is retrieved to form FMM images.

For FMM, the equivalent excitation rate is proportional to the temporal change in the intensity of the ballistic component:

$$
\begin{aligned}
E_{\mathrm{FMM}}\left(\mathbf{r}_{F}, \mathbf{r}_{M}\right)= & \mathrm{e}^{-\mu_{l} z_{M} C}\left\{\left|\int_{0}^{1} J_{0}(v \xi) \mathrm{e}^{i u \xi^{2} / 2} \xi d \xi\right|^{2}\right. \\
& -\mid \int_{0}^{0.707} J_{0}(v \xi) \mathrm{e}^{i u \xi^{2} / 2} \xi d \xi \\
& \left.-\left.\int_{0.707}^{0} J_{0}(v \xi) \mathrm{e}^{i u \xi^{2} / 2} \xi d \xi\right|^{2}\right\}
\end{aligned}
$$

The first term in Eq. (4.5) is the maximal excitation intensity when $H(\mathbf{r}, t)=\phi_{0}$, and the second term corresponds to the minimum excitation intensity when $H\left(\mathbf{r} \notin R_{2}, t\right)=\phi_{0}+\pi$.

The excitation point spread function (PSF) are compared in Fig. 5. The focal point is around $400 \mu \mathrm{m}$ (or $4 l_{s}$ ) below the medium surface in this example. The equivalent PSF for FMM (Fig. 5(a)) is diffraction limited. For CM, the ballistic excitation rate (Fig. 5(b)) is also diffraction limited. However, the scattered excitation rate is widely distributed in the medium (Fig. 5(c)) and is the main source of background.

FMM and CM share the same detection light path so the detection PSF is identical for both methods. It is essentially the difference in the excitation PSF that leads to significantly improved background rejection in FMM. As shown in Fig. 6, the SBR for FMM decreases moderately by less than $13 \mathrm{~dB}$ when the imaging depth increases from $100 \mu \mathrm{m}$ to $900 \mu \mathrm{m}$. It is evident that FMM can provide a respectable 
(a)

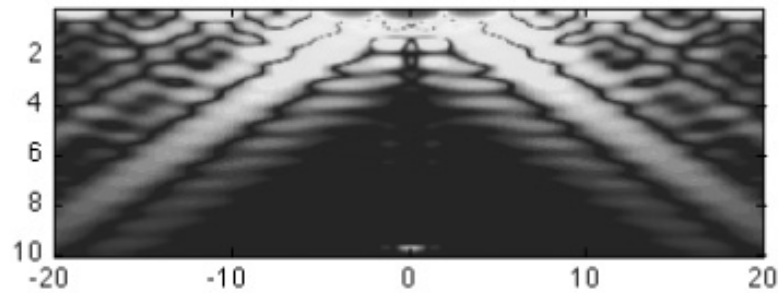

(b)

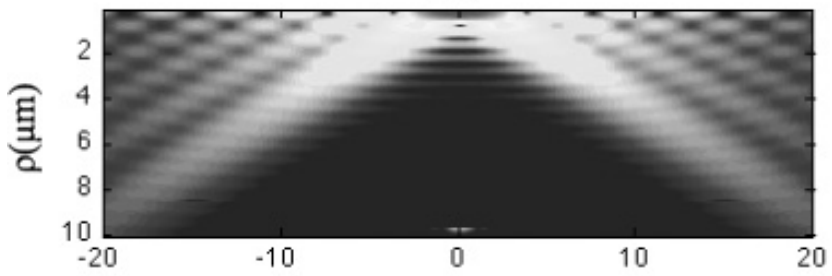

(c)

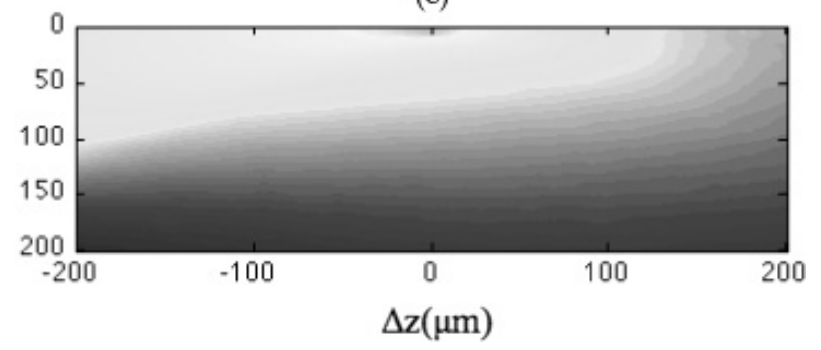

Fig. 5. (a) FMM equivalent PSF compared with (b) the ballistic excitation rate and (c) scattered excitation rate of $\mathrm{CM}$.

SBR even for an imaging depth over $1 \mathrm{~mm}$. This is in sharp contrast to CM, for which the SBR decays rapidly with increasing depth. The SBR for CM falls below $0 \mathrm{~dB}$ for an imaging depth beyond $250 \mu \mathrm{m}$, which is a typical penetration depth for CM.

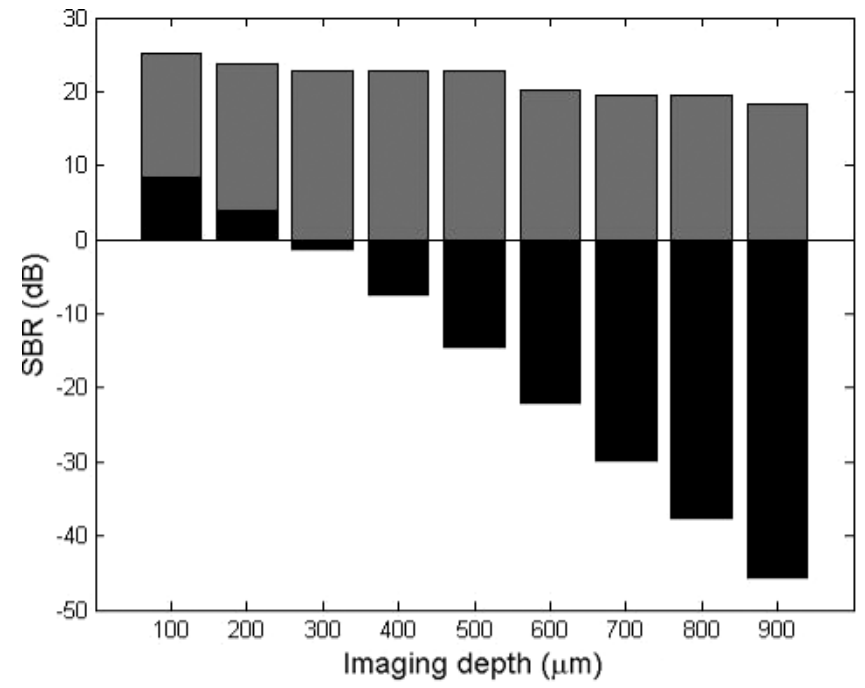

Fig. 6. SBR of FMM (circles) and CM (asterisks) as a function of imaging depth.
Another important issue in FMM is the SNR. The noise floor is fundamentally limited by shot noise, which depends on the magnitude of the dc component in the emission light captured by the pinhole detector. The FMM signal intensity, on the other hand, is determined by the amplitude of the ac component.

The modulation depth in FMM is defined as

$$
M=\frac{I_{\mathrm{ac}}}{I_{\mathrm{dc}}} .
$$

The dc component $I_{\mathrm{dc}}$ is proportional to the mean power of excitation light delivered to the sample. In practical situations, the excitation power needs to be maintained at the lowest possible level to reduce photobleaching and photo toxicity. In addition, $I_{\mathrm{dc}}$ contributes to shot noise, which cannot be completely eliminated by filtering. Consequently, it is desirable to maximize the modulation depth $M$ for the best SNR. This can be achieved by optimal design of the STPM, or more specifically, the aperture configuration. ${ }^{194}$

Illustrated in Fig. 7 are a few aperture configurations that are relatively inexpensive to fabricate. The relative phase delay $\varphi(t)$ between the white and dark regions varies from 0 to $\pi$. All the apertures are so designed that the total area of the white regions is equal to that of the dark regions. Figure 8 shows the simulation results of effective illumination PSFs

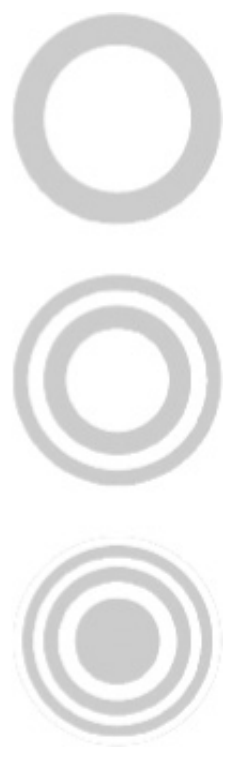

(a)
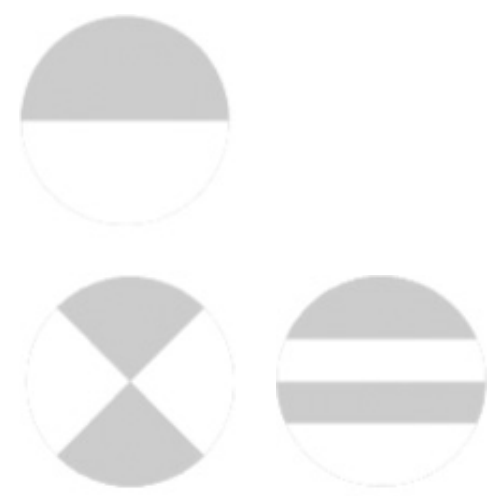

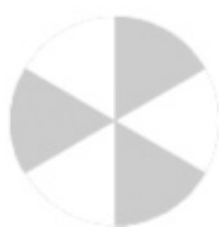

(b)

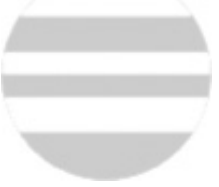

(c)
Fig. 7. Annular (a), fan-shaped (b), and stripe-shaped (c) apertures. 

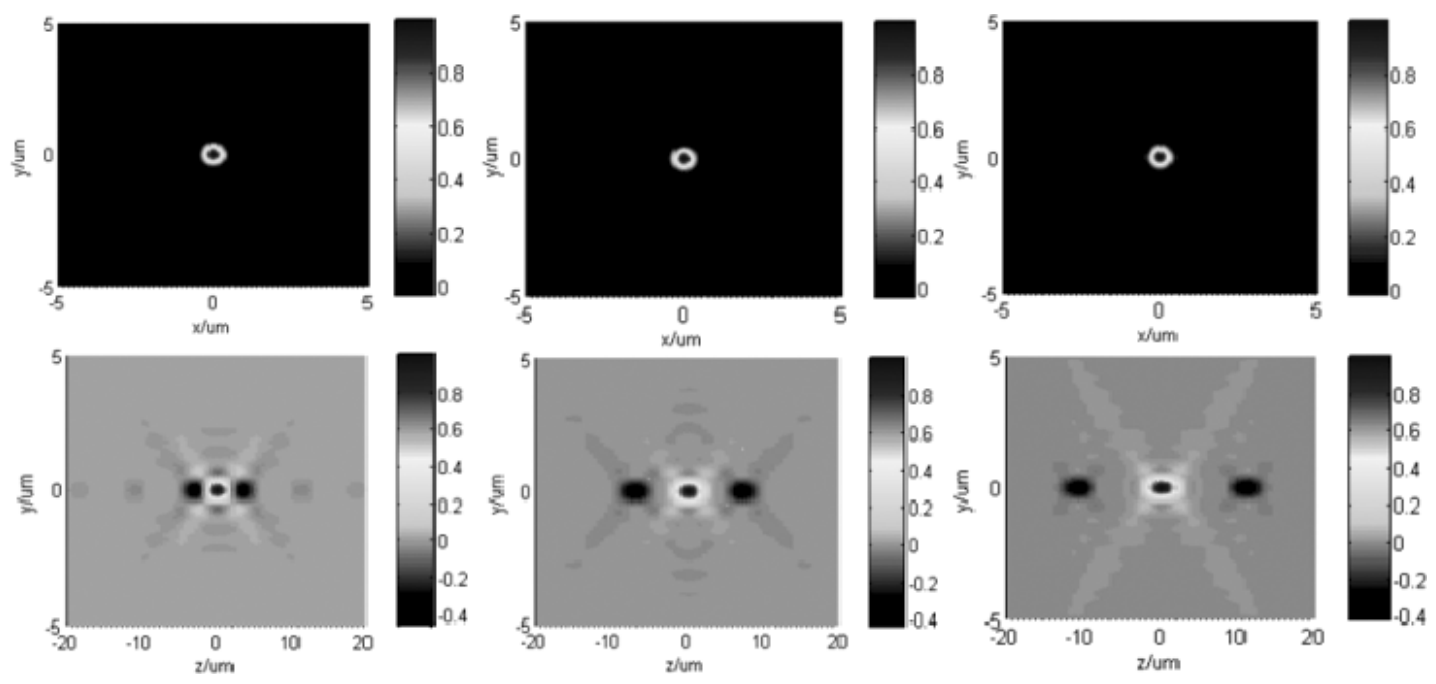

Fig. 8. Effective illumination PSFs for annular apertures.

Table 1. Modulation depth.

\begin{tabular}{cccc}
\hline Number of zones & Annular & Fan-shaped & Stripe-shaped \\
\hline 2 & 0.4395 & 0.3525 & 0.3525 \\
4 & 0.7175 & 0.5746 & 0.6199 \\
6 & 0.8228 & 0.6948 & 0.7353 \\
\hline
\end{tabular}

for annular apertures of two zones (Fig. 7(a)), four zones (Fig. 7(b)), and six zones (Fig. 7(c)). Distributions in the focal plane $(X-Y)$ are provided in the top row while the cross-sectional distributions are included in the bottom row.

The modulation depths are summarized in Table 1. Generally the modulation depth tends to increase with an increasing number of zones. However, the improvement from four-zones to six-zones becomes less significant. In addition, the annular aperture is the best among all, and the stripe shape is better than the fan shape.

\subsection{Design of spatiotemporal phase modulator}

As revealed by theoretical studies, the STPM design has a profound impact on the performance of FMM. Over the past few years, several versions of STPM havebeen proposed and reported.

The first ever FMM system was based on a very simple STPM consisting of two mirrors with parallel reflective surfaces. ${ }^{192}$ Each mirror reflects half of the illumination beam. One of them is mounted on a stationary base, while another is glued on a PZT actuator. The PZT actuator is driven by a periodic voltage signal so as to introduce a time-dependent phase shift. There are two major problems related to this simple implementation. First of all, the resonant frequency of PZT actuators are usually less than tens of $\mathrm{kHz}$, which fundamentally limits the minimum signal acquisition time per pixel to the order of $1 \mathrm{~ms}$. This is too slow in comparison with most confocal microscopes. Second, the parallelism of the two mirrors is difficult to maintain over long periods of time.

The tilting plate phase modulator successfully solved the parallelism problem. ${ }^{195}$ Its structure is shown in Fig. 9. A glass plate mounted on a galvanometer is inserted into the optical path, bisecting the light beam in half. This subjects half of the optical beam to a phase delay determined by the tilting angle, while the remaining portion remains unperturbed. Given that the glass plate surfaces are parallel and homogenous, the two half beams can be kept parallel to one another. However, such an implementation of STPM is still limited by the resonant frequency of the galvanometer.

Acousto-optic modulators (AOMs) are known to be able to provide frequency shifts of up to a few hundred MHz. Nevertheless, the commercially available AOMs do not provide spatially separated modulation of the illumination beam. A high-speed STPM (Fig. 10) that includes two AOMs was proposed in 2010. ${ }^{196}$

The coherent illumination beam from the laser is first split by a beam splitter into two sub-beams that then pass through two AOMs (M080-2B/F-GH2) with slightly different resonance frequencies (i.e. $f_{1}$ 


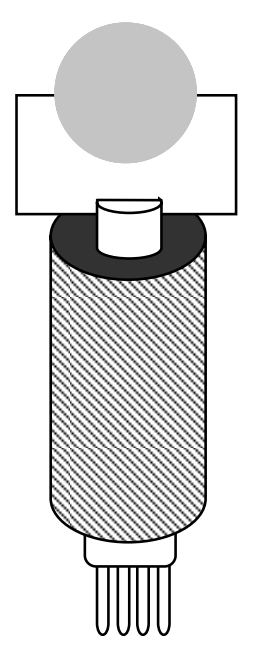

(a)

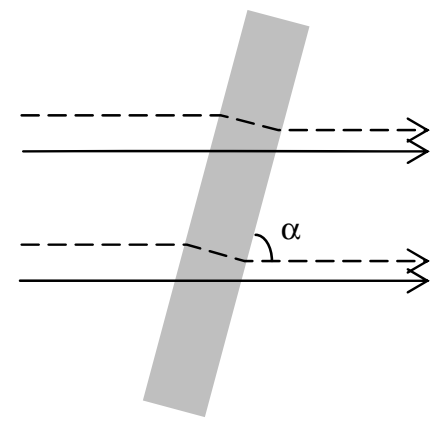

(b)
Fig. 9. (a) Glass plate mounted on a galvanometer, intersecting half of the excitation beam whose cross section is indicated by the gray circle. (b) Beams passing (solid lines) or by passing (dashed lines) the glass plate have an optical path length difference that is tilting angle dependent.

and $f_{2}$ ). The first-order diffracted sub-beams are Doppler shifted by $f_{1}$ and $f_{2}$ on each pass. They are reflected with a slight lateral displacement using retro-reflectors to return to the same AOMs and then are recombined at beam splitter, when the Doppler frequency shifts are doubled. Part of the combined beam is directed towards a fiber-optic photodetector, which generates a reference signal at the optical beat frequency of $2\left(f_{1}-f_{2}\right)$. The remaining part of the modulated laser beam is directed to the scanning unit of a conventional CM (e.g., Olympus FV300). In the

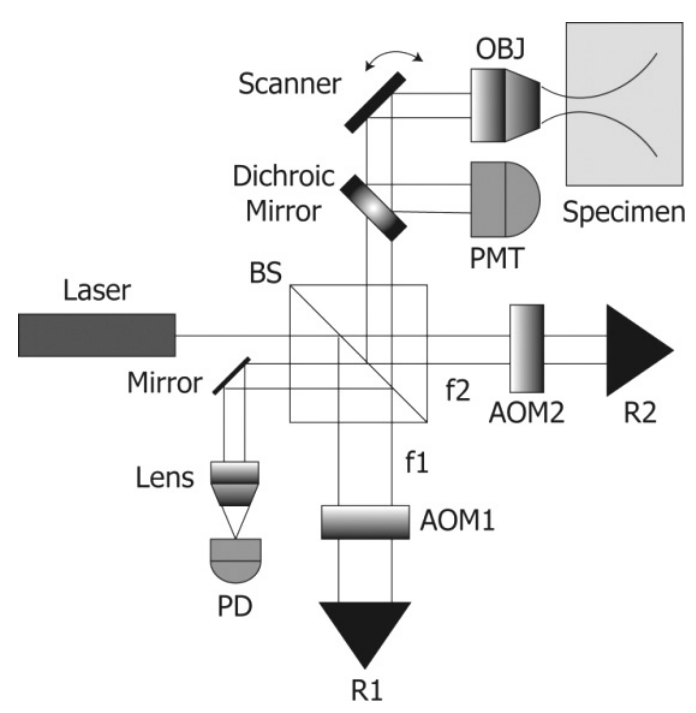

Fig. 10. A STPM comprised of two AOMs. setup reported in Ref. 196, the resonant frequencies of the two AOMs are 75 and $80 \mathrm{MHz}$, respectively, resulting in a FMM modulation frequency of $10 \mathrm{MHz}$ in the fluorescence emission. The $10-\mathrm{MHz}$ modulation frequency is adequately high for demodulating the FMM signal and forming FMM images in real time. In principle, one can remove one the AOMs so that the corresponding sub-beam is not frequency shifted. Consequently, the FMM modulation frequency will be greater than $100 \mathrm{MHz}$. While this may be useful for fluorescence lifetime imaging, it can introduce additional signal attenuation, depending on the PMT time response and the lifetime of the fluorescence molecules.

While AOM-based STPM provides a viable approach for real-time acquisition of FMM images, there are two major technical drawbacks. First, AOMs are wavelength-dependent devices. Optical realignment is required when switching the illumination laser wavelength. Second, the two sub-beams do not fill the entire aperture and the resultant modulation depth $(\sim 0.25)$ is nonideal.

The latest STPM design is based on EOM or electro-optical modulator, which is a high-speed phase modulation device. ${ }^{191}$ An EOM does not alter the light propagation direction. However, its phase modulation is polarization dependent. Figure 11(a) shows the design of a STPM. The major components of the STPM include an EOM and a few polarization optical components. The excitation light enters the EOM with its polarization at $45^{\circ}$ with the modulation axis $(Y)$ of the EOM driven by a high frequency $\left(f_{0}=9.85 \mathrm{MHz}\right)$ sine wave signal. The horizontal component of the excitation beam is phase modulated at $f_{0}$ while the vertical component (of equal power) is subject to a constant phase delay. The EOM is followed by a spatial polarizer for spatial separation of the modulated and the nonmodulated excitation light. An example implementation of the spatial polarizer is shown in Fig. 11(b). It selectively passes vertically polarized (nonmodulated) or horizontally polarized (modulated) excitation beam. The second polarizer P2 following the spatial polarizer projects the modulated and nonmodulated fields on to the same polarization axis so that they can interfere with each other when converging to the focal point.

The EOM-based STPM enjoys many advantages such as high-speed, flexible aperture configuration (enabling high modulation depth), and compatibility with multiple excitation wavelengths. 


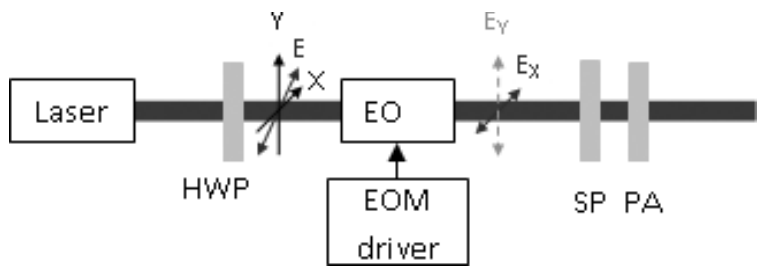

(a)

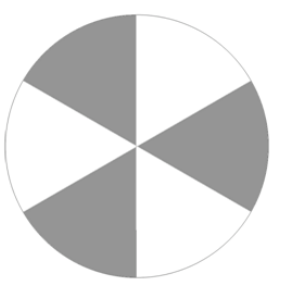

(b)

Fig. 11. (a) A STPM is inserted in the excitation light path of an Olympus FV300 confocal microscope for FMM imaging. (b) A six-zone spatial polarizer that selectively passes horizontally polarized light (white zones) or vertically polarized light (gray zones). HWP: Half wave plate; SP: spatial polarizer; PA: polarization analyzer.

\subsection{Imaging performances}

The main advantage of FMM over conventional $\mathrm{CM}$ is the additional background rejection mechanism offered by focal modulation, which can enhance the signal to background ratio and imaging depth. ${ }^{193,197}$ Other than imaging depth, it has been found that FMM also helps improve the spatial resolution. ${ }^{198,199}$ The improvement in depth imaging can be explained by noting that the excitation beams cross only in the focal region, similar to the methods of angular gating discussed in Sec. 1.5. ${ }^{61-63}$ Hence optical sectioning results from a combination of confocal and angular gating. However, unlike the divided aperture system, where the two sub-apertures are in the illumination and detection paths, the two sub-apertures are both in the illumination path. The coherence of the illumination of the two sub-apertures means that the resolution is determined by the size of the complete aperture. Actually, spatial resolution can be better than in CM, because the system can be thought of as a scanning structured illumination microscope. ${ }^{198}$

One of the advantages of FMM over established deep imaging microscopy methods, i.e., multi-photon microscopy and OCM, is its compatibility with multiple contrast mechanisms. MPM is compatible with fluorescence only, while OCM can only detect reflectance or backscattering. FMM, on the other hand, is compatible with most contrast mechanisms including single-photon fluorescence, two-photon fluorescence, and reflectance/scattering.

Multi-contrast imaging of biological sample was demonstrated using the latest FMM system. ${ }^{191}$ FMM fluorescence/scattering images (tilted 3D projection) of an Epipremnum Aureum leaf are shown in Fig. 12. The leaf was placed in a glassbottom dish and its top surface was facing down against a glass coverslip. Distilled water was added to the dish for refractive index matching. The microscope was operated in the XYZ mode for acquiring 3D image stacks from 71 to $150 \mu \mathrm{m}$. The image acquisition speed was $8 \mu$ s per pixel. A 40x/ 1.1 water immersion objective lens (441857-9970000, Carl Zeiss) was used in the imaging experiments. The red channel corresponds to autofluorescence from chloroplasts (excited by a $488 \mathrm{~nm}$

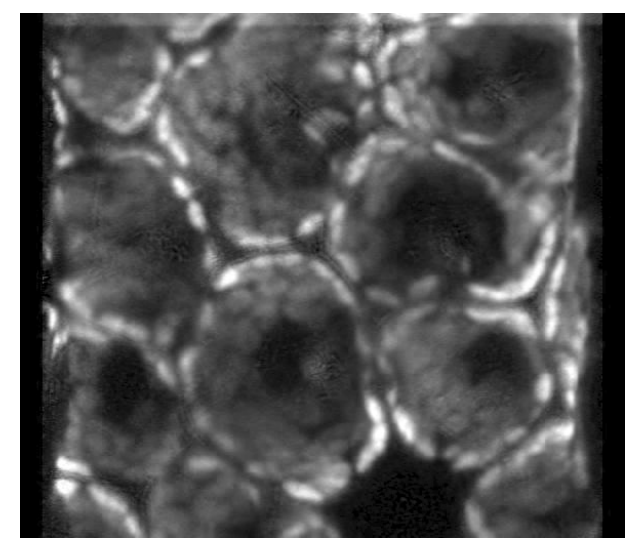

(a)

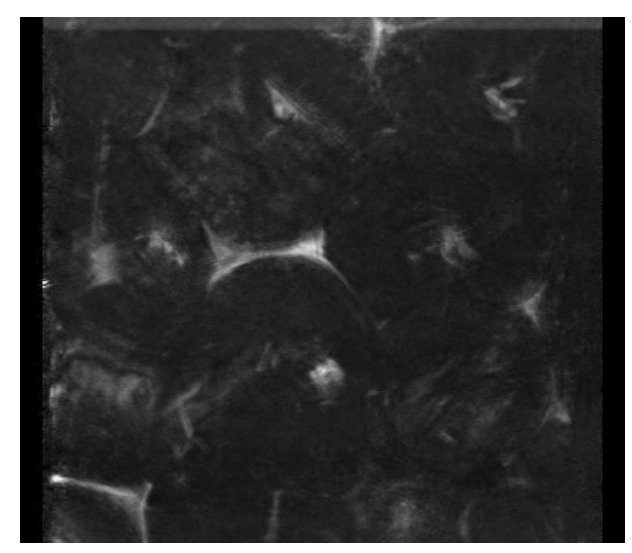

(b)

Fig. 12. FMM fluorescence (a) and scattering (b) images. 
blue laser) and the green channel is related to cell membranes and intracellular organelles that scatter or reflect the excitation light at $559 \mathrm{~nm}$. It is obvious that the sub-cellular features revealed by both contrast mechanisms were perfectly aligned.

Quantitative comparisons of background rejection were made using a tissue phantom as the sample, in which $\mathrm{TiO}_{2}$ nanoparticles are included as microscopic scatterers. ${ }^{196}$ An Olympus LUCPLANFLN 20x/0.45 NA objective was used to obtain the backscattering images. Figures 13(a) and 13(b) are CM and FMM images, respectively, at a depth of $320 \mu \mathrm{m}$. The FMM image appears to have structures more clearly distinguishable. The plot at the bottom of each image is the intensity profile along the white line. It is evident that the background level in the CM image is elevated, more noticeably within the cluster.
For the region of interest indicated by the square boxes in Fig. 13, spectral analysis was performed for both CM and FMM images. One can see from Fig. 14 that the high frequency components $(>1 \mathrm{cycle} / \mu \mathrm{m})$ in the FMMimage are about $13 \mathrm{~dB}$ stronger than those in the CM image. This can be translated to a $13 \mathrm{~dB}$ improvement in the signal to background ratio provided by FMM.

Surprisingly, the best FMM imaging depth in biological tissue was reported in the first paper on FMM. ${ }^{192}$ Chicken cartilage was used as the sample tissue. Chondrocytes were stained with DiD (DiIC18(5), Invitrogen Corp.), a lipophilic tracer for cell membrane labeling. CM and FMM images were obtained using the first prototype FMM system. CM image quality became unacceptable after the imaging depth was greater than $300 \mu \mathrm{m}$. However, the FMM image at $500 \mu \mathrm{m}$ depth (Fig. 15(a))
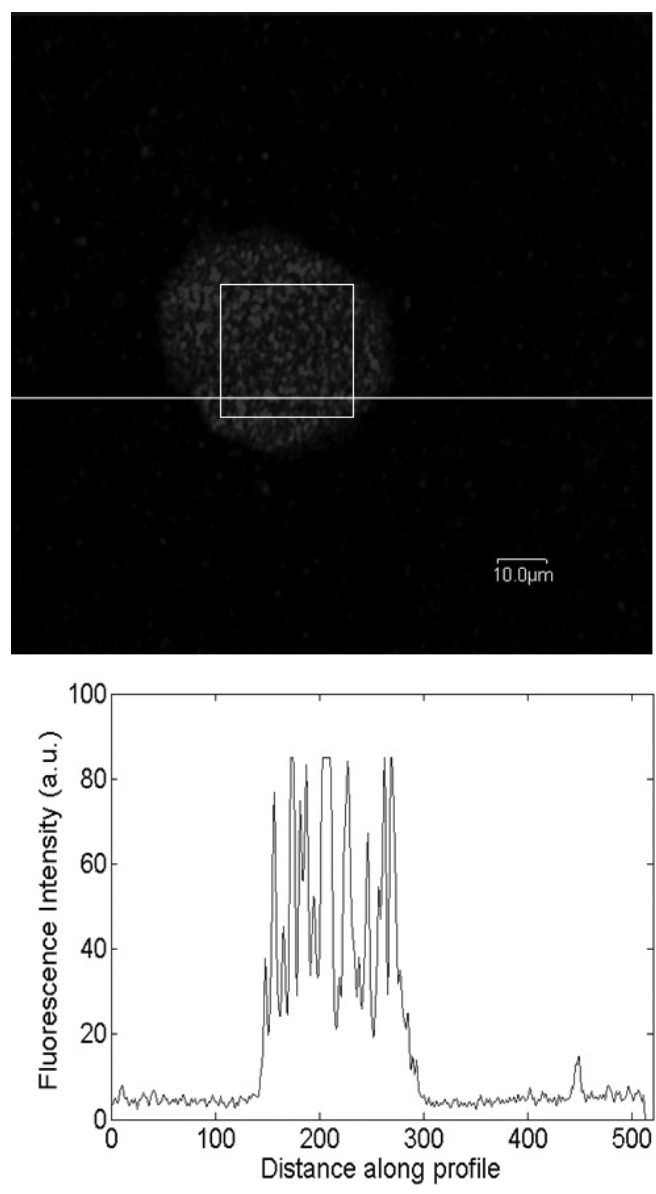

(a)
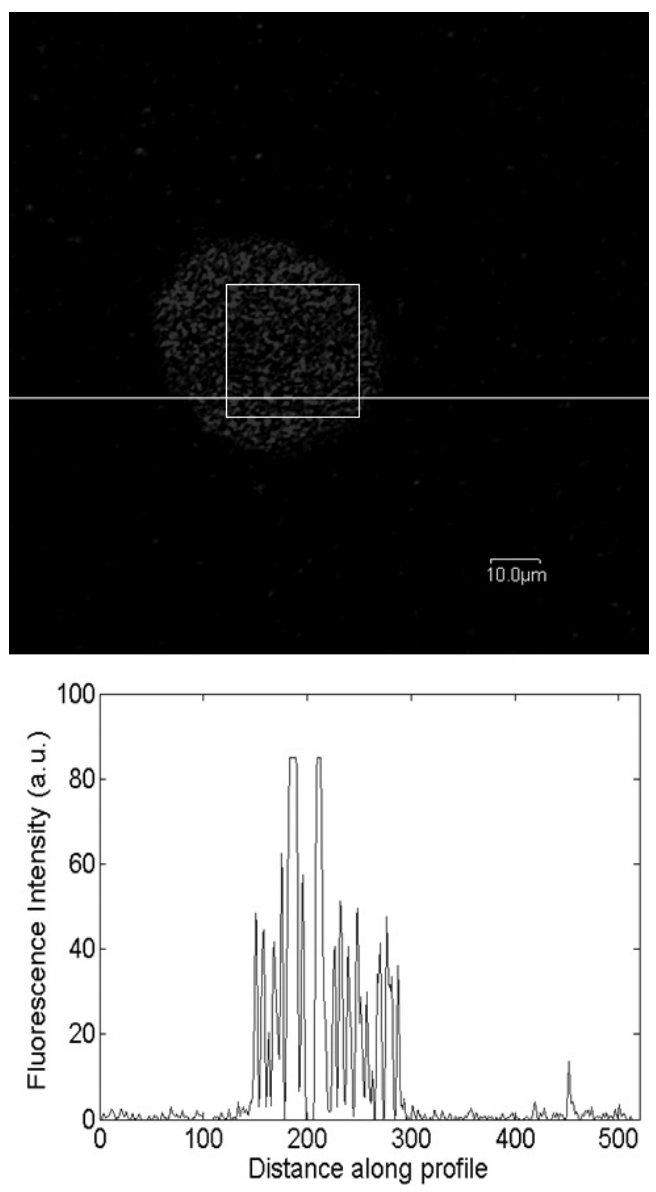

(b)

Fig. 13. (a) $\mathrm{CM}$ and (b) FMM images of cluster of $\mathrm{TiO}_{2}$ nanoparticles. The plot at the bottom of the images shows the intensity profile of the white lines in the images. Peaks are more distinct with better contrast can be observed. 


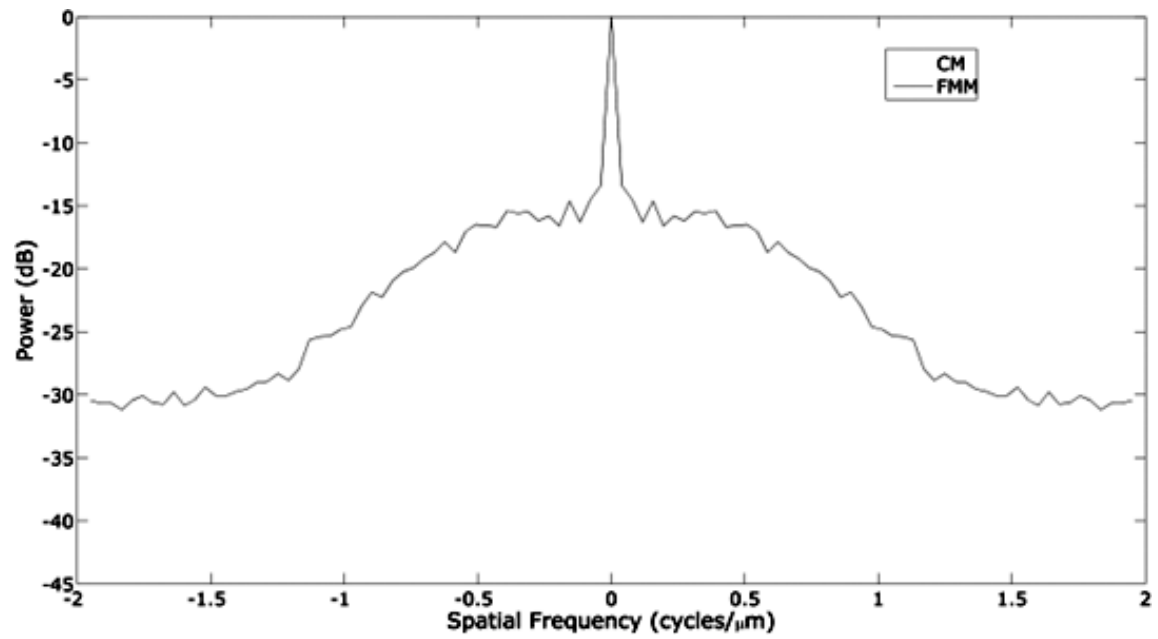

(a)

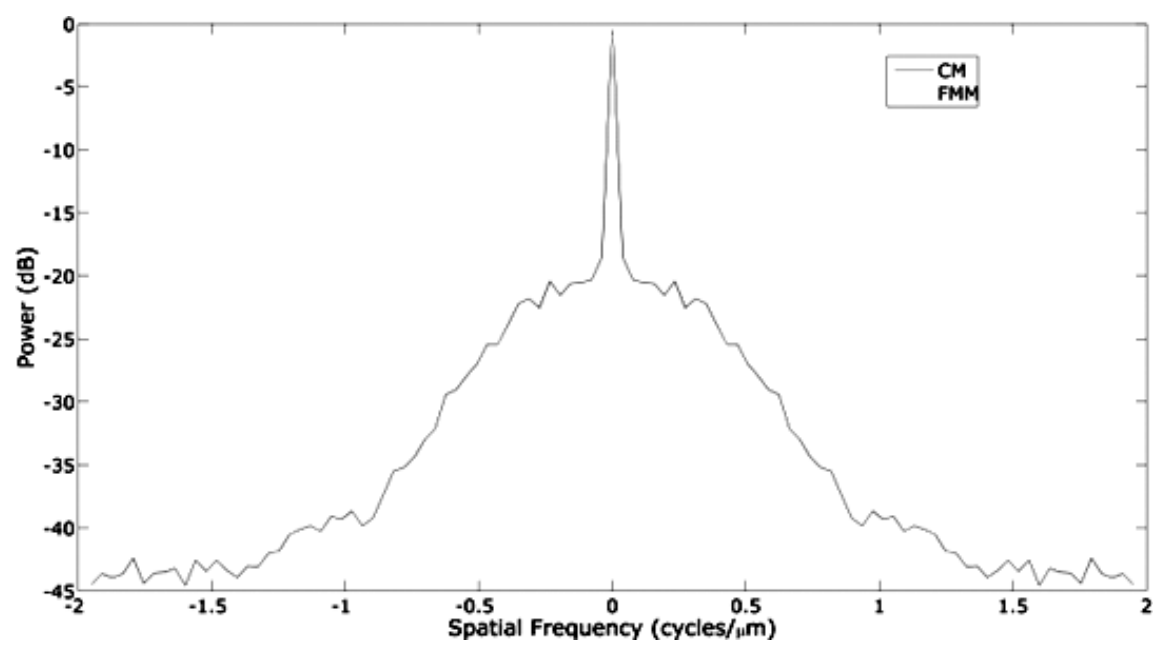

(b)

Fig. 14. Normalized spatial spectra in cycle/ $\mu \mathrm{m}$ of the region of interests labeled by square boxes in Fig. 13(a) and 13(b) showing the spatial frequency components of (a) CM and (b) FMM respectively.

provided detailed information with sub-micron spatial resolution and excellent contrast. It was still possible to visualize cellular structures around $600 \mu \mathrm{m}$ (Fig. 15(b)). Beyond $600 \mu \mathrm{m}$ the shot noise associated with the background started to overwhelm the FMM signal. It should be noted that the image acquisition time was relatively long for FMM images shown in Fig. 15. This was due to the low modulation frequency $(\sim 5 \mathrm{kHz})$ of the dual-mirror SPTM.

\subsection{Two-photon FMM}

Two-photon excitation has unique characteristics that make it very attractive for biomedical imaging, especially in thick samples. ${ }^{200,201}$ The imaging depth of two-photon microscopy is fundamentally limited by the onset of out-of-focus fluorescence generated near the top surface of the sample. ${ }^{202}$ One way to increase the penetration depth is simply to increase the laser average power. However, increasing the laser average power can lead to saturation of the excitation, photobleaching, and photodamage. Other methods include reducing the laser repetition rate $^{203}$ and using longer excitation wavelengths. ${ }^{129,131-133}$ Recently, Leray and Mertz developed a differential aberration two-photon microscopy using a deformable mirror to further reject out-of-focus background. However, because of the limited frame rate, subtraction cannot fully 


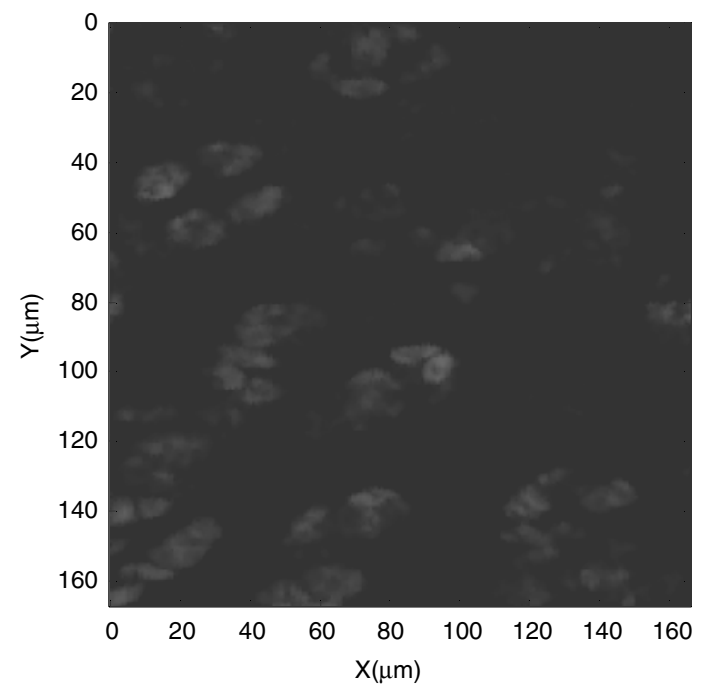

(a)

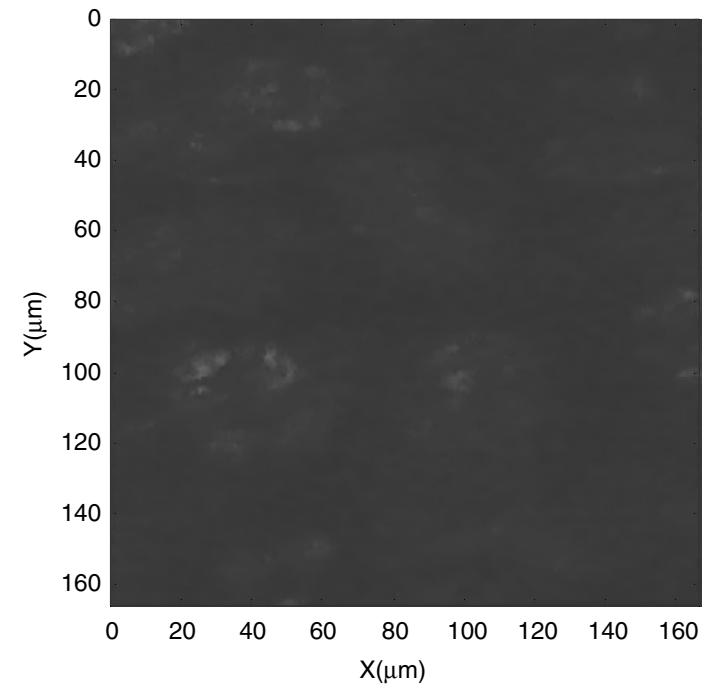

(b)

Fig. 15. FMM images obtained from (a) 500 microns and (b) 600 microns in depth.

eliminate the background. ${ }^{204}$ This limitation can be overcome by the focal modulation technique, which has been discussed in detail in previous sections.

Theoretical study has been carried out to investigate the feasibility of two-photon focal modulation microscopy (2PFMM). It was based on a similar theoretical model reported in Ref. 193, except the nonlinear dependency of excitation rate on light intensity. ${ }^{205}$ Figure 16 shows the SBR of $2 \mathrm{PFMM}$ versus $2 \mathrm{P}$ fluorescence microscopy, as a function of focus depth $z_{0}$. When the focal depth is less than $3 l_{s}$, the SBR of either 2PFMM or conventional twophoton fluorescence microscopy is almost constant. However, the SBR is approximately $7 \mathrm{~dB}$ higher in 2PFMM than that in 2PM. When the focal depth is larger than $3 l_{s}$, the $\mathrm{SBR}$ in conventional $2 \mathrm{P}$

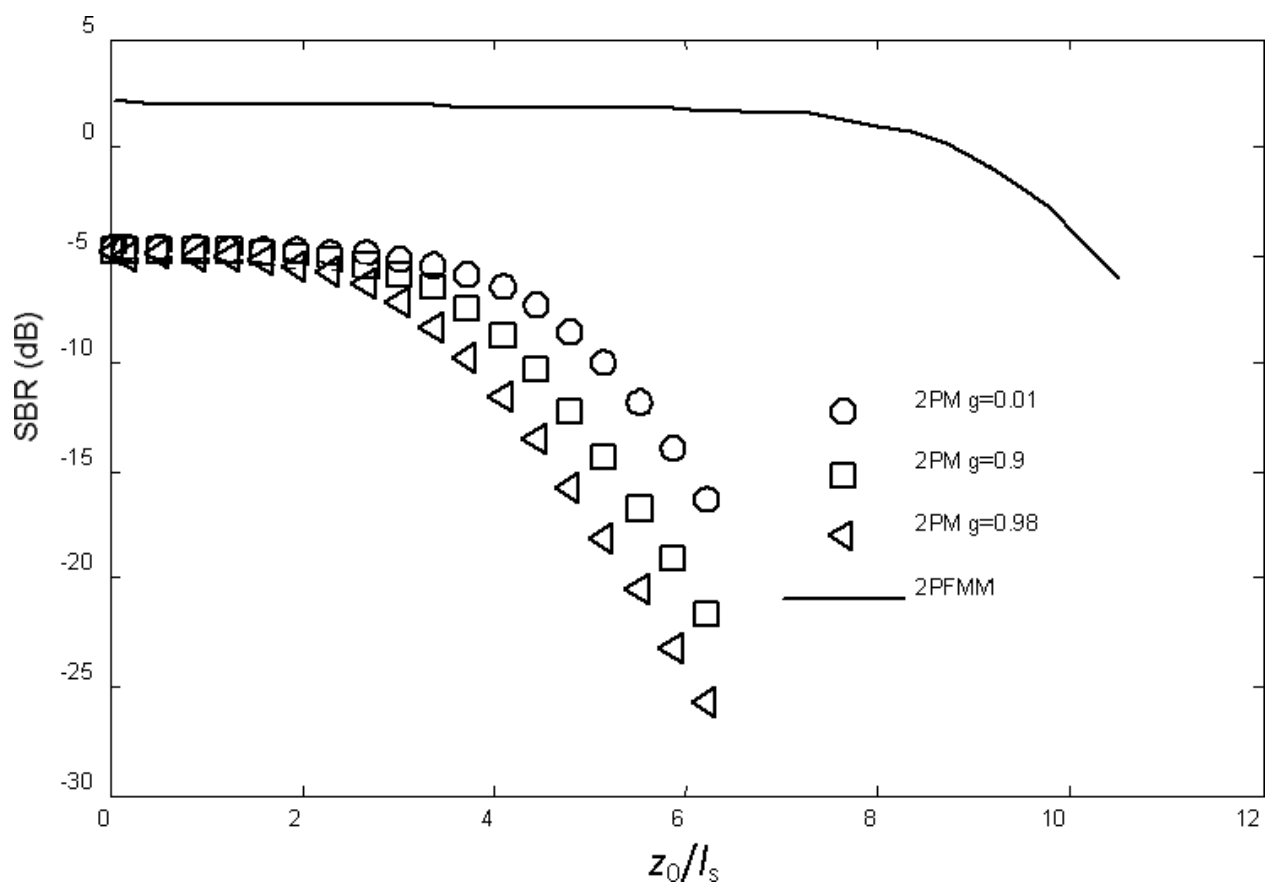

Fig. 16. The signal to background ratio of $2 \mathrm{PFMM}$ and $2 \mathrm{PM}$ as a function of focal depth $z_{0}$. Simulation parameters are: $l_{s}=200 \mu \mathrm{m}, n=1.33, N A=5 / 9$, and $\lambda=0.9 \mu \mathrm{m}$. 
fluorescence microscopy begins to decay significantly as the near-surface background fluorescence and scattered light comes into play. In contrast, the SBR in 2PFMM remains nearly constant until the focal depth reaches $7 l_{s}$. This indicates that the scattered-light excited fluorescence plays a less important role to the background in 2PFMM. If the imaging depth is SBR limited, it can be improved by a factor of 2-3 (depending on the anisotropy factor $g$ ) by integrating focal modulation into a twophoton microscope.

\section{Conclusion and Outlook}

MPM and OCM are well established as deep tissue imaging methods. However, there have been considerable efforts to further enhance their performance. Future prospects for OCM and MPM lie in the development of higher power and ultra-broadband light sources, new interferometric configurations, and faster scanning techniques. FMM is a relatively new imaging modality and has the potential to become a useful tool for a wide range of biomedical imaging applications.

Adaptive optics will play a significant role in future development of imaging techniques for highly scattering media and deeper imaging through thick samples. Aberrations induced by the sample are universal problems that affect all types of light microscopes. However, the effects are most detrimental when the imaging depth goes beyond a few layers of cells, leading to low contrast and poor resolution. The best imaging depth of FMM has been demonstrated with chicken cartilage, which is relatively more homogeneous than other tissue types. It is, therefore of great importance to correct for the aberrations so that the optimal performance of deep tissue imaging can be obtained. There are still many challenges that prevent adaptive optics from being successfully incorporated as a useful feature into microscope to correct for aberrations. While real-time adaptive optics for FMM and twophoton microscopes is desirable, it would only be possible with improved aberration measurement schemes and faster correction devices.

\section{References}

1. W.-F. Cheong, S. A. Prahl, A. J. Welch, "A review of the optical properties of biological tissue," IEEE J. Quantum Electron. 26, 2166-2185 (1990).
2. Y. Ueno, M. Shimizu, "An optical-fiber cable fault location method," IEEE J. Quantum Electron. 75, 77D-78D (1975).

3. M. K. Barnoski, S. M. Jensen, "Fibre waveguides: A novel technique for investigating attenuation characteristics," Appl. Opt. 15, 2112-2115 (1976).

4. F. G. Fujimoto, S. De Silvestri, E. P. Ippen, C. A. Puliafito, R. Margolis, A. Oseroff, "Femtosecond optical ranging in biological systems," Opt. Lett. 11, 150-152 (1986).

5. K. M. Yoo, R. R. Alfano, "Time-resolved coherent and incoherent components of forward light scattering in random media," Opt. Lett. 15, 320-322 (1990).

6. S. L. Jacques, "Time resolved propagation of ultrashort laser pulses within turbid tissue," Appl. Opt. 28, 2223-2229 (1989).

7. S. Anderson-Engels, R. Berg, O. Jarlmann, S. Svanberg, "Time- resolved transillumination for medical diagnostics," Opt. Lett. 15, 1179-1181 (1990).

8. D. G. Papaioannou, G. W. Hooft, J. J. M. Baselman, M. J. C. van Gemert, "Image quality in timeresolved transillumination of highly scattering medium," Appl. Opt. 34, 6144-6157 (1995).

9. R. I. MacDonald, "Frequency domain optical reflectometry," Appl. Opt. 20, 1840-1844 (1981).

10. W. Eickhoff, R. Ulrich, "Optical frequency-domain reflectometry in single-mode fiber," Appl. Phys. Lett. 39, 693-695 (1981).

11. M. Davidson, K. Kaufman, I. Mazor, F. Cohen, "An application of interference microscopy to integrated circuit inspection and metrology," Proc. SPIE, Vol. 775, pp. 233-247 (1987).

12. E. Beaurepaire, A.-C. Boccara, M. Lebec, L. Blanchot, H. Saint-Jalmes, "Full-field optical coherence tomography," Opt. Lett. 23, 244-246 (1998).

13. D. Huang, E. A. Swanson, C. P. Lin, J. S. Schuman, W. G. Stinson, W. Chang, M. R. Lee, T. Flotte, K. Gregory, C. A. Puliafito, J. G. Fujimoto, "Optical coherence tomography," Science 254, 1178-1181 (1991).

14. C. J. R. Sheppard, T. Wilson, "Effects of high angles of convergence on $V(z)$ in the scanning acoustic microscope," App. Phys. Lett. 38, 858-859 (1981).

15. D. K. Hamilton, T. Wilson, C. J. R. Sheppard, "Experimental observations of the depth-discrimination properties of scanning microscopes," Opt. Lett. 6, 625-626 (1981).

16. I. J. Cox, "Scanning optical fluorescence microscopy," J. Microsc. 133, 149-154 (1984).

17. C. J. Cogswell, C. J. R. Sheppard, "Imaging using confocal brightfield techniques," Institute of Physics Conference Series 98, 633-638 (1990). 
18. C. J. Cogswell, D. K. Hamilton, C. J. R. Sheppard, "Colour reflection microscopy using red, green and blue lasers," J. Microsc. 165, 103-117 (1992).

19. C. J. R. Sheppard, T. Wilson, "Depth of field in the scanning microscope," Opt. Lett. 3, 115-117 (1978).

20. J. M. Schmitt, A. Kniittel, M. Yadlowsky, "Confocal microscopy in turbid media," J. Opt. Soc. Am. A 11, 2226-2235 (1994).

21. M. Minsky, "Microscopy apparatus," US Patent 3,013,467 filed 7 November, 1957 (1961).

22. Z. Koana, J. Illumination Eng. Inst. 26, 371-385 (1942).

23. H. Naora, "Microspectrophotometry and cytochemical analysis of nucleic acids," Science 114, 279-280 (1951).

24. C. J. R. Sheppard, A. Choudhury, "Image formation in the scanning microscope," Opt. Acta 24, 1051-1073 (1977).

25. C. J. R. Sheppard, X. Mao, "Confocal microscopes with slit apertures," J. Mod. Opt. 35, 1169-1185 (1988).

26. R. Wolleschensky, B. Zimmermann, "High-speed confocal fluorescence imaging with a novel line scanning microscope," J. Biomed. Opt. 11, 064011 (2006).

27. H. Goldman, "Spaltlampenphotographie undphotometrie," Ophthalmologica 98, 257-270 (1940).

28. M. Petran, M. Hadravsky, D. Egger, R. Galambos, "Tandem scanning reflected light microscope," J. Opt. Soc. Am. 58, 661-664 (1968).

29. M. D. Egger, M. Petran, "New reflected-light microscope for viewing unstained brain and ganglion cells," Science 305-307 (1967).

30. C. J. R. Sheppard, T. Wilson, "The theory of the direct-view confocal microscope," J. Microsc. 124, 107-117 (1981).

31. C. J. R. Sheppard, T. Wilson, "Image formation in scanning microscopes with partially coherent source and detector," Opt. Acta 25, 315-325 (1978).

32. T. Wilson, A. R. Carlini, "Size of detector in confocal imaging systems," Opt. Lett. 12, 227-229 (1987).

33. M. Gu, C. J. R. Sheppard, "Three-dimensional imaging in confocal fluorescent microscopy with annular lenses," J. Mod. Opt. 38, 2247-2263 (1991).

34. M. Gu, C. J. R. Sheppard, "Confocal fluorescent microscopy with a finite-sized circular detector," $J$. Opt. Soc. Am. A 9, 151-153 (1992).

35. M. Gu, T. Tannous, C. J. R. Sheppard, "Effect of numerical aperture, pinhole size and annular pupil on confocal imaging through highly scattering media," Opt. Lett. 21, 312-314 (1996).
36. M. Kempe, A. Z. Genack, W. Rudolph, P. Dorn, "Ballistic and diffuse light detection in confocal and heterodyne imaging systems," J. Opt. Soc. Am. A 14, 216-223 (1997).

37. X. S. Gan, S. P. Schilders, M. Gu, "Image formation in turbid media under a microscope," J. Opt. Soc. Am. A 15, 2052-2058 (1998).

38. L. Giniunas, R. Juskaitis, S. V. Shatalin, "Scanning fiber-optic microscope," Electron. Letters 27, 724-726 (1991).

39. T. Dabbs, M. Glass, "Single-mode fibers used as confocal microscope pinholes," Appl. Opt. 31, 705706 (1992).

40. P. M. Delaney, M. H. Harris, R. G. King, "Fibreoptic laser scanning confocal microscopy suitable for fluorescence imaging," Appl. Opt. 33, 573-577 (1994).

41. M. Gu, C. J. R. Sheppard, "Signal level of the fibre optical confocal scanning microscope," J. Mod. Opt. 38, 1621-1630 (1991).

42. M. Gu, C. J. R. Sheppard, "Axial resolution in the fibre-optical confocal scanning microscope using annular lenses," Opt. Commun. 88, 27-32 (1992).

43. W. Lukosz, M. Marchand, "Optischen Abbildung unter Überschreitung der Beugungsbedingten Auflösungsgrenze," Opt. Acta 10, 241-255 (1963).

44. M. M. A. Neil, R. Juskaitis, T. Wilson, "Method for obtaining optical sectioning by using structured light in a conventional microscope," Opt. Lett. 22, 1905-1907 (1997).

45. M. G. L. Gustafsson, D. A. Agard, J. W. Sedat, "I5M: 3D widefield light microscopy with better than 100nm axial resolution," J. Microsc. 195, 10-16 (1999).

46. M. Gustafsson, "Surpassing the lateral resolution limit by a factor of two using structured illumination microscopy," J. Microsc. 198, 82-87 (2000).

47. V. J. Corcoran, "Directional characteristics in optical heterodyne detection processes," J. Appl. Phys. 36, 1819-1825 (1965).

48. Y. Fujii, H. Takimoto, "Imaging properties due to the optical heterodyne and its application to laser microscopy," Opt. Comm. 18, 45-47 (1976).

49. T. Sawatari, "Optical heterodyne scanning microscope," Appl. Opt. 12, 2768-2772 (1973).

50. M. Kempe, W. Rudolph, "Scanning microscopy through thick layers based on linear correlation," Opt. Lett. 19, 1919-1921 (1994).

51. M. Kempe, W. Rudolf, "Comparative study of confocal and heterodyne microscopy for imaging through scattering media," J. Opt. Soc. Am. A 13, 46-52 (1996).

52. J. A. Izatt, M. R. Hee, G. M. Owen, E. A. Swanson, J. G. Fujimoto, "Optical coherence microscopy in scattering media," Opt. Lett. 19, 590-592 (1994). 
53. H. Siedentopf, R. Zsigmondy, "Uber Sichtbarmachung und Grössenbestimmung ultramikroskopischer Teilchen, mit besonderer Anwendung auf Goldrubingläser," Ann. der Phys. 10, 1-39 (1903).

54. D. M. Maurice, "Cellular membrane activity in the corneal endothelium of the intact eye," Experientia 15, 1094-1095 (1968).

55. D. M. Maurice, "A scanning slit optical microscope," Invest. Ophthalmol. 13, 1033-1037 (1974).

56. C. J. Koester, "A scanning mirror microscope with optical sectioning characteristics: Applications in ophthalmology," Appl. Opt. 19, 1749-1757 (1980).

57. C. J. Koester, "Comparison of optical sectioning methods: The scanning slit confocal microscope," in Handbook of Confocal Microscopy, J. Pawley, Ed., Plenum Press, New York, 525-533 (1990).

58. P. J. Dwyer, C. A. DiMarzio, "Confocal reflectance theta line scanning microscope for imaging human skin in vivo," Opt. Lett. 31, 942-944 (2006).

59. P. J. Dwyer, C. A. DiMarzio, M. Rajadhyaksha, "Confocal theta line-scanning microscope for imaging human tissues," Appl. Opt. 46, 1843-1851 (2007).

60. C. J. R. Sheppard, M. D. Sharma, "Integrated intensity, and imaging through scattering media," J. Mod. Opt. 48, 1517-1525 (2001).

61. C. J. R. Sheppard, W. Gong, K. Si, "The divided aperture technique for microscopy through scattering media," Opt. Exp. 33, 1599-1602 (2008).

62. W. Gong, K. Si, C. J. R. Sheppard, "Optimization of axial resolution in confocal microscope with D-shaped apertures," Appl. Opt. 48, 3998-4002 (2009).

63. W. Gong, K. Si, C. J. R. Sheppard, "Divided-aperture technique for fluorescence confocal microscopy through scattering media," Appl. Opt. 49, 752-757 (2010).

64. E. H. K. Stelzer, S. Lindek, S. Albrecht, R. Pick, G. Ritter, N. J. Salmon, R. Stricker, "A new tool for the observation of embryos and other large specimens - confocal theta-fluorescence microscopy," J. Microsc. 179, 1-10 (1995).

65. R. H. Webb, F. Rogomentich, "Confocal microscope with large field and working distance," Appl. Opt. 38, 4870-4875 (1999).

66. T. D. Wang, M. J. Mandella, C. H. Contag, G. S. Kino, "Dual-axis confocal microscope for highresolution in vivo imaging," Opt. Lett. 28, 414-416 (2003).

67. T. D. Wang, C. H. Contag, M. J. Mandella, N. Y. Chan, G. S. Kino, "Dual-axes confocal microscopy with post-objective scanning and low-coherence heterodyne detection," Opt. Lett. 28, 1915-1917 (2003).
68. J. T. C. Liu, M. J. Mandella, S. Friedland, R. Soetikno, J. H. Crawford, C. H. Contag, G. S. Kino, T. D. Wang, "Dual-axes confocal reflectance microscope for distinguishing colonic neoplasia," J. Biomed. Opt. 11, 054019 (2006).

69. L. K. Wong, M. J. Mandella, G. S. Kino, T. D. Wang, "Improved rejection of multiply scattered photons in confocal microscopy using dual-axes architecture," Opt. Lett. 32, 1674-1676 (2007).

70. C. J. R. Sheppard, M. Gu, "Optical sectioning in confocal microscopes with annular pupil," Optik 86, 169-172 (1991).

71. C. J. R. Sheppard, M. Gu, "Improvement of axial resolution in confocal microscopy using an annular pupil," Opt. Commun. 84, 7-13 (1991).

72. M. Gu, C. J. R. Sheppard, H. Zhou, "Optimization of axial resolution in confocal imaging using annular pupils," Optik 93, 87-90 (1993).

73. X. S. Gan, S. P. Schilders, M. Gu, "Combination of annular aperture and polarization gating methods for efficient microscopic imaging through turbid medium: Theoretical analysis," Microsc. Anal. 3, 495-503 (1997).

74. M. Gu, C. J. R. Sheppard, "Effects of annular pupils on confocal fluorescent imaging," J. Mod. Opt. 39, 1883-1896 (1992).

75. M. Gu, T. Tannous, C. J. R. Sheppard, "Improved axial resolution in confocal fluorescence microscopy using annular pupils," Opt. Commun. 110, 533539 (1994).

76. A. H. Voie, "Three-dimensional reconstruction of the cochlear from two-dimensional images of optical sections," Comput. Med. Imag. Graph. 19, 377-384 (1995).

77. J. Huisken, J. Swoger, F. Del Bene, J. Wittbrodt, E. H. K. Stelzer, "Optical sectioning deep inside live embryos by selective plane illumination microscopy," Science 305, 1007-1009 (2004).

78. C. J. Engelbrecht, E. H. K. Stelzer, "Resolution enhancement in a light-sheet-based microscope (SPIM)," Opt. Lett. 31, 1477-1479 (2006).

79. J. Huisken, D. Y. R. Stainier, "Even fluorescence excitation by multidirectional selective plane illumination microscopy (mSPIM)," Opt. Lett. 32, 2608-2610 (2007).

80. H.-U. Dodt, U. Leischner, A. Schierloh, N. Jähling, C. Mauch, K. Deininger, J. M. Deussing, M. Eder, W. Zieglgänsberger, K. Becker, "Ultramicroscopy: Three-dimensional visualization of neuronal networks in the whole mouse brain," Nat. Meth. 4, 331-336 (2007).

81. T. Breuninger, K. Greger, E. H. K. Stelzer, "Lateral modulation boosts image quality in single plane illumination fluorescence microscopy," Opt. Lett. 32, 1938-1940 (2007). 
82. F. O. Fahrbach, P. Simon, A. Rohrbach, "Microscopy with self-reconstructing beams," Nat. Photon. 4, 780-785 (2010).

83. F. O. Fahrbach, A. Rohrbach, "A line scanned light-sheet microscope with phase shaped selfreconstructing beams," Opt. Exp. 18, 24229-24244 (2010).

84. T. A. Planchon, L. Gao, D. E. Milkie, M. W. Davidson, J. A. Galbraith, C. G. Galbraith, E. Betzig, "Rapid three-dimensional isotropic imaging of living cells using Bessel beam plane illumination," Nat. Meth. 8, 417-423 (2011).

85. S. Kalchmair, N. Jaerling, K. Becker, H.-U. Dodt, "Image contrast enhancement in confocal ultramicroscopy," Opt. Lett. 35, 79-81 (2010).

86. C. Tjokro, C. J. R. Sheppard, "Photon scattering phase space analysis in multiplanes within a microscope system," Proc. SPIE, Vol. 6163, U1630 (2006).

87. C. Tjokro, C. J. R. Sheppard, "Effects of apertures on scattered light: A Monte Carlo study of confocal imaging," Proc. SPIE, Vol. 6536, G5350 (2007).

88. H. Horinaka, K. Hashimoto, K. Wada, Y. Cho, M. Osawa, "Extraction of quasi-straightforwardpropagating photons from diffused light transmitting through a scattering medium by polarization modulation," Opt. Lett. 20, 1501-1503 (1995).

89. K. M. Yoo, R. R. Alfano, "Time resolved depolarization of multiple backscattering light from random media," Phys. Lett. A 142, 531-536 (1989).

90. X. S. Gan, S. P. Schilders, M. Gu, "Image enhancement through turbid media under a microscope by use of polarization gating methods," J. Opt. Soc. Am. A 16, 2177-2184 (1999).

91. C. J. R. Sheppard, R. Kompfner, "Resonant scanning optical microscope," Appl. Opt. 17, 28792882 (1978).

92. R. Hellwarth, P. Christiansen, "Nonlinear optical microscopic examination of structure in polycrystalline ZnSe," Opt. Commun. 12, 312-322 (1974).

93. R. Hellwarth, P. Christiansen, "Nonlinear optical microscope using second harmonic generation," Appl. Opt. 14, 247-248 (1975).

94. C. J. R. Sheppard, R. Kompfner, J. Gannaway, D. Walsh, "The scanning harmonic optical microscope," IEEE J. Quantum Electron. 13, 100D (1977).

95. J. Gannaway, C. J. R. Sheppard, "Second harmonic imaging in the scanning optical microscope," Opt. Quantum Electron. 10, 435-439 (1978).

96. I. Freund, M. Deutsch, "Second-harmonic microscopy of biological tissue," Opt. Lett. 11, 94-96 (1986).

97. I. Freund, M. Deutsch, A. Sprecher, "Connective tissue polarity, optical second harmonic microscopy, crossed-beam summation and small-angle scattering in rat-tail tendon," Biophys. J. 50, 693-712 (1986).

98. M. D. Duncan, J. Reintjes, T. J. Manuccia, "Scanning coherent anti-Stokes Raman microscope," Opt. Lett. 7 (1982).

99. W. Denk, J. H. Strickler, W. W. Webb, "Twophoton laser scanning fluorescence microscopy," Science 248, 73-76 (1990).

100. W. Denk, J. P. Strickler, W. W. Webb, "Twophoton laser microscopy," US Patent 5034613 (1991).

101. M. Muller, J. Squier, K. R. Wilson, G. J. Brakenhoff, "3D microscopy of transparent objects using third-harmonic generation," J. Microsc. 191, 266274 (1998).

102. S. W. Hell, K. Bahlmann, M. Schrader, M. Soini, H. Malak, I. Gryczynski, J. R. Lakowicz, "Threephoton excitation in fluorescence microscopy," $J$. Biomed. Opt. 1, 71-74 (1996).

103. C. J. R. Sheppard, "Image formation in threephoton fluorescence microscopy," Bioimaging 4, 124-128 (1996).

104. M. Gu, "Resolution in three-photon fluorescence scanning microscopy," Opt. Lett. 21, 988-990 (1996).

105. R. Gauderon, P. B. Lukins, C. J. R. Sheppard, "Simultaneous multi-channel two-photon microscopy," Micron 32, 685-689 (2001).

106. M. Gu, C. J. R. Sheppard, "Comparison of threedimensional imaging properties between twophoton and single-photon fluorescence microscopy," J. Microsc. 177(2), 128-137 (1995).

107. X. S. Gan, M. Gu, "Confocal fluorescence can rely on chemically or biologically specific fluorescent labels, or intrinsic autofluorescence," J. Appl. Phys. 87, 3214-3221 (2000).

108. M. Gu, C. J. R. Sheppard, "Effects of a finite-sized pinhole on 3-D image formation in confocal twophoton fluorescence microscopy," J. Mod. Opt. 40, 2009-2024 (1993).

109. R. Gauderon, C. J. R. Sheppard, "Improvement in imaging in confocal fluorescent microscopes using detector arrays," Bioimaging 6, 126-129 (1998).

110. R. Gauderon, P. B. Lukins, C. J. R. Sheppard, "Effect of a confocal pinhole in two-photon microscopy," Microsc. Res. Tech. 47, 210-214 (1999).

111. R. Gauderon, C. J. R. Sheppard, "Effect of a finitesize pinhole on the noise performance in single-, two- and three-photon fluorescence microscopy," Appl. Opt. 38, 3562-3565 (1999).

112. P. Török, C. J. R. Sheppard, "The role of pinhole size in high-aperture two- and three-photon microscopy," in Confocal and Two-Photon 
Microscopy: Foundations, Applications and Advances, A. Diaspro, Ed., pp. 127-152, WileyLiss, New York (2002).

113. S. Maiti, J. B. Shear, R. M. Williams, W. R. Zipfel, W. W. Webb, "Measuring serotonin distribution in live cells with three-photon excitation," Science 275, 530-532 (1997).

114. M. Schrader, K. Bahlmann, S. W. Hell, "Threephoton-excitation microscopy: Theory, experiment and applications," Optik 104, 116-124 (1997).

115. P. J. Campagnola, M.-d. Wei, A. Lewis, L. M. Loew, "High-resolution nonlinear optical imaging of live cells by second harmonic generation," Biophys. J. 77, 3341-3349 (1999).

116. R. Gauderon, P. B. Lukins, C. J. R. Sheppard, "Three-dimensional second-harmonic generation imaging with femtosecond laser pulses," Opt. Lett. 23, 1209-1211 (1998).

117. J. N. Gannaway, C. J. R. Sheppard, "Second-harmonic imaging in the scanning optical microscope," Opt. Quantum Electron. 10, 435-439 (1978).

118. C. J. R. Sheppard, R. Kompfner, "Resonant scanning optical microscope," Appl. Opt. 17, 28792882 (1978).

119. M. D. Duncan, J. Reintjes, T. J. Manuccia, "Scanning coherent anti-Stokes Raman microscope," Opt. Lett. 7, 350 (1982).

120. Y. Barad, H. Eisenberg, M. Horowitz, Y. Silberberg, "Nonlinear scanning laser microscopy by third-harmonic generation," App. Phys. Lett. 70, 922-924 (1997).

121. M. Müller, J. Squier, K. R. Wilson, G. J. Brakenhoff, "3D-microscopy of transparent objects using third-harmonic generation," J. Microsc. 191, 266274 (1998).

122. J. A. Squier, M. Muller, G. J. Brakenhoff, "Third harmonic generation microscopy," Opt. Exp. 3, 315-324 (1998).

123. D. Yelin, Y. Silberberg, "Laser scanning thirdharmonic-generation microscopy in biology," Opt. Exp. 5, 169-175 (1999).

124. P. Theer, M. T. Hasan, W. Denk, "Two-photon imaging to a depth of $1000 \mu \mathrm{m}$ in living brains by use of a Ti: $\mathrm{Al}_{2} \mathrm{O}_{3}$ regenerative amplifier," Opt. Lett. 28, 1022-1024 (2003).

125. M. Müller, J. Squier, R. Wolleschensky, U. Simon, G. J. Brakenhoff, "Dispersion pre-compensation of 15 femtosecond optical pulses for high-numericalaperture objectives," J. Microsc. 191, 141-150 (1998).

126. S. Sakadzic, U. Demirbas, T. R. Mempel, A. Moore, S. Ruvinskaya, D. A. Boas, A. Sennaroglu, F. X. Kaertner, J. G. Fujimoto, "Multi-photon microscopy with a low-cost and highly efficient $\mathrm{Cr}$ : licaf laser," Opt. Exp. 16, 20848-20863 (2008).
127. S.-W. Chu, I.-H. Chen, T.-M. Liu, P. C. Chen, C.-K. Sun, B.-L. Lin, "Multimodal nonlinear spectral microscopy based on a femtosecond $\mathrm{Cr}$ : forsterite laser," Opt. Lett. 26, 1909-1911 (2001).

128. S.-W. Chu, S. Chen, T. Tsai, T.-M. Liu, C. P. Lin, H. Tsai, C.-K. Sun, "In vivo developmental biology study using noninvasive multi-harmonic generation microscopy," Opt. Exp. 11, 3093-3099 (2003).

129. M. Balu, T. Baldacchini, J. Carter, T. B. Krasieva, R. Zadoyan, B. J. Tromberg, "Effect of excitation wavelength on penetration depth in nonlinear optical microscopy of turbid media," J. Biomed. Opt. 14, 010508 (2009).

130. T. Yasui, Y. Takahashi, M. Ito, S. Fukushima, T. Araki, "Ex vivo and in vivo second-harmonic generation imaging of dermal collagen fiber in skin: Comparison of imaging characteristics between modelocked Cr:forsterite and Ti:sapphire lasers," Appl. Opt. 48, D88-D95 (2009).

131. D. Kobat, M. E. Durst, N. Nishimura, A. W. Wong, C. B. Schaffer, C. Xu, "Deep tissue multiphoton microscopy using longer wavelength excitation," Opt. Exp. 17, 13354-13364 (2009).

132. D. Kobat, N. G. Horton, C. Xu, "In vivo twophoton microscopy to $1.6-\mathrm{mm}$ depth in mouse cortex," J. Biomed. Opt. 16, 106014 (2011).

133. N. G. Horton, K. Wang, D. Kobat, C. G. Clark, F. W. Wise, C. B. Schaffer, C. Xu, "In vivo threephoton microscopy of subcortical structures within an intact mouse brain," Nat. Photon. 7, 205-209 (2013).

134. J. Bewersdorf, R. Pick, S. W. Hell, "Multifocal multi-photon microscopy," Opt. Lett. 23, 655-657 (1998).

135. A. H. Buist, M. Müller, J. Squier, G. J. Brakenhoff, "Real time two photon absorption microscopy using multipoint excitation," J. Microsc. 192, 217-226 (1998).

136. J. Qu, L. Liu, Y. Shao, H. Niu, B. Z. Gao, "Recent progress in multifocal multiphoton microscopy," J. Innovative Opt. Health Sci. 5, 1250018 (2012).

137. H. Kim, C. Buehler, P. T. C. So, "High-speed twophoton scanning microscope," Appl. Opt. 38, 6004-6009 (1999).

138. G. Y. Fan, H. Fujisaki, A. Miyawaki, R.-K. Tsay, R. Y. Tsien, M. H. Ellisman, "Video-rate scanning two-photon excitation fluorescence microscopy and ratio imaging with Cameleons," Biophys. J. 78, 2412-2420 (1999).

139. V. Iyer, B. E. Losavio, P. Saggau, "Compensation of spatial and temporal dispersion for acousto-optic multiphoton laser-scanning microscopy," $J$. Biomed. Opt. 8, 460-471 (2003).

140. R. Kurtz, M. Fricke, J. Kalb, P. Tinnefeld, M. Sauer, "Application of multiline two-photon 
microscopy to functional in vivo imaging," $J$. Neurosci. Methods 151, 276-286 (2006).

141. K. Bahlmann, P. T. So, M. Kirber, R. Reich, B. Kosicki, W. McGonagle, K. Bellve, "Multifocal multiphoton microscopy (MMM) at a frame rate beyond $600 \mathrm{~Hz}$," Opt. Exp. 15, 10991-10998 (2007).

142. K. H. Kim, C. Buehler, K. Bahlmann, T. Ragan, W.-C. A. Lee, E. Nedivi, E. L. Heffer, S. Fantini, P. T. C. So, "Multifocal multiphoton microscopy based on multianode photomultiplier tubes," Opt. Exp. 15, 11658-11678 (2007).

143. M. Kempe, W. Rudolph, "Impact of chromatic and spherical aberration on the focusing of ultrashort light pulses by lenses," Opt. Lett. 18, 137-139 (1993).

144. E. Papagiakoumou, V. de Sars, V. Emiliani, D. Oron, "Temporal focusing with spatially modulated excitation," Opt. Exp. 17, 5391-5401 (2009).

145. H. Dana, S. Shoham, "Numerical evaluation of temporal focusing characteristics in transparent and scattering media," Opt. Exp. 14, 4937-4948 (2011).

146. D. Oron, Y. Silberberg, "Spatiotemporal coherent control using shaped, temporally focused pulses," Opt. Exp. 13, 9903-9908 (2005).

147. G. Zhu, J. van Howe, M. Durst, W. Zipfel, C. Xu, "Simultaneous spatial and temporal focusing of femtosecond pulses," Opt. Exp. 13, 2153-2159 (2005).

148. D. J. McCabe, A. Tajalli, D. R. Austin, P. Bondareff, I. A. Walmsley, S. Gigan, B. Chatel, "Spatio-temporal focusing of an ultrafast pulse through a multiply scattering medium," Nat. Commun. 2, 447 (2011).

149. R. Hellwarth, P. Christensen, "Nonlinear optical microscopic examination of structure in polycrystalline ZnSe," Opt. Commun. 12, 318-322 (1974).

150. S.-W. Chu, S.-P. Tai, M.-C. Chan, C.-K. Sun, I. C. Hsiao, C.-H. Lin, Y.-C. Chen, B.-L. Lin, "Thickness dependence of optical second harmonic generation in collagen fibrils," Opt. Exp. 15, 12005-12010 (2007).

151. I. Freund, M. Deutsch, A. Sprecher, "Connective tissue polarity: Optical second-harmonic microscopy, crossed-beam summation, and small-angle scattering in rat-tail tendon," Biophys. J. 50, 693-712 (1986).

152. P. J. Campagnola, A. C. Millard, M. Terasaki, P. E. Hoppe, C. J. Malone, W. A. Mohler, "Threedimensional high-resolution second-harmonic generation imaging of endogenous structural proteins in biological tissues," Biophys. J. 82, 493-508 (2002).
153. S. J. Lin, C. Y. Hsiao, Y. Sun, W. Lo, W. C. Lin, G. J. Jan, S. H. Jee, C. Y. Dong, "Monitoring the thermally induced structural transitions of collagen by use of second-harmonic generation microscopy," Opt. Lett. 30, 622-624 (2005).

154. V. Barzda, C. Greenhalgh, J. Aus der Au, Elmore S, J. van Beek, J. Squier, "Visualization of mitochondria in cardiomyocytes by simultaneous harmonic generation and fluorescence microscopy," Opt. Exp. 13, 8263-8276 (2005).

155. G. Mizutani, Y. Sonoda, H. Sano, M. Sakamoto, T. Takahashi, S. Ushioda, "Detection of starch granules in a living plant by optical second harmonic microscopy," J. Luminescence 87-89, 824$826(2000)$.

156. G. Cox, N. Moreno, J. Feijó, "Second-harmonic imaging of plant polysaccharides," J. Biomed. Opt. 10, 0240131-0240136 (2005).

157. N. Prent, R. Cisek, C. Greenhalgh, R. Sparrow, N. Rohitlall, M. S. Milkereit, C. Green, V. Barzda, "Application of nonlinear icroscopy for studying the structure and dynamics in biological systems," Proc. SPIE Vol. 5971, 597106 (2005).

158. L. Moreaux, O. Sandre, M. Blanchard-Desce, J. Mertz, "Membrane imaging by simultaneous second-harmonic generation and two-photon microscopy," Opt. Lett. 25, 320-322 (2000).

159. S.-W. Chu, I.-H. Chen, T.-M. Liu, C.-K. Sun, S.-P. Lee, B.-L. Lin, P.-C. Cheng, M.-X. Kuo, D.-J. Lin, H.-L. Liu, "Nonlinear bio-photonic crystal effects revealed with multimodal nonlinear microscopy," J. Microsc. 208, 190-200 (2002).

160. T. Y. F. Tsang, "Optical third-harmonic generation at interfaces," Phys. Rev. A 52, 4116-4125 (1995).

161. J. A. Squier, M. Muller, G. J. Brakenhoff, K. R. Wilson, "Third harmonic generation microscopy," Opt. Exp. 3, 315-324 (1998).

162. D. Oron, E. Tal, Y. Silberberg, "Depth-resolved multiphoton polarization microscopy by third-harmonic generation," Opt. Lett. 28, 2315-2317 (2003).

163. D. Oron, D. Yelin, E. Tal, S. Raz, R. Fachima, Y. Silberberg, "Depth-resolved structural imaging by third-harmonic generation microscopy," J. Struct. Biol. 147, 3-11 (2004).

164. V. Shcheslavskiy, G. I. Petrov, S. Saltiel, V. V. Yakovlev, "Quantitative characterization of aqueous solutions probed by the third-harmonic generation microscopy," J. Struct. Biol. 147, 42-49 (2004).

165. D. Débarre, N. Olivier, E. Beaurepaire, "Signal epidetection in third-harmonic generation microscopy of turbid media," Opt. Exp. 15, 8913-8924 (2007). 
166. E. J. Gualda, G. Filippidis, G. Voglis, M. Mari, C. Fotakis, N. Tavernarakis, "In vivo imaging of cellular structures in Caenorhabditis elegans by combined TPEF, SHG and THG microscopy," J. Microsc. 229, 141-150 (2008).

167. A. Zumbusch, G. R. Holtom, X. S. Xie, "Three dimensional vibrational imaging by coherent antiStokes Raman scattering," Phys. Rev. Lett. 82, 4142-4145 (1999).

168. Y. R. Shen, The Principles of Nonlinear Optics (Wiley, New York 1984).

169. J. A. Izatt, M. R. Hee, G. M. Owen, E. A. Swanson, J. G. Fujimoto, "Optical coherence microscopy in scattering media," Opt. Lett. 19, 590-592 (1994).

170. J. A. Izatt, M. D. Kulkarni, H.-W. Wang, K. Kobayashi, M. V. Sivak, "Optical Coherence Tomography and Microscopy in Gastrointestinal Tissues," IEEE J. Sel. Topics Quantum Electron. 2, 1017-1028 (1996).

171. T. Li, A. Wang, K. Murphy, R. Claus, "Whitelight scanning for michelson interferometer for absolute position-distance measurement," Opt. Lett. 20, 785-787 (1995).

172. H. Maruyama, S. Inoue, T. Mitsuyama, M. Ohmi, M. Haruna, "Low-coherence interferometer system for the simultaneous measurement of refractive index and thickness," Appl. Opt. 41, 1315-1322 (2002).

173. E. A. Swanson, D. Huang, M. R. Hee, J. G. Fujimoto, C. P. Lin, C. A. Puliafito, "High-speed optical coherence domain reflectometry," Opt. Lett. 17, 151-153 (1992).

174. A. G. Podoleanu, G. M. Dobre, D. A. Jackson, "En-face coherence imaging using galvanometer scanner modulation," Opt. Lett. 23, 147-149 (1998).

175. G. J. Tearney, B. E. Bouma, S. A. Boppart, B. Golubovic, E. A. Swanson, J. G. Fujimoto, "Rapid acquisition of in vivo biological images by use of optical coherence tomography," Opt. Lett. 21, 1408-1410 (1996).

176. A. M. Weiner, J. P. Heritage, E. M. Kirschner, "High-resolution femtosecond pulse shaping," $J$. Opt. Soc. Am. B 5, 1563-1572 (1988).

177. A. M. Rollins, S. Yazdanfar, M. Kulkarni, R. UngArunyawee, J. A. Izatt, "In vivo video rate optical coherence tomography," Opt. Exp. 3, 219-229 (1998).

178. G. J. Tearney, B. E. Bouma, J. G. Fujimoto, "High-speed phase- and group-delay scanning with a grating-based phase control delay line," Opt. Lett. 22, 1811-1813 (1997).

179. C. J. R. Sheppard, S. S. Kou, C. Depeursinge, "Reconstruction in interferometric synthetic aperture microscopy: Comparison with optical coherence tomography and digital holographic microscopy," J. Opt. Soc. Am. A 29, 244-250 (2012).

180. M. Gu, X. Gan, C. J. R. Sheppard, "Threedimensional coherent transfer functions in fibre optical confocal scanning microscopes," J. Opt. Soc. Am. A 8, 1019-1025 (1991).

181. M. Villiger, T. Lasser, "Image formation and tomogram reconstruction in optical coherence microscopy," J. Opt. Soc. Am. A 27, 2216-2228 (2010).

182. C. J. R. Sheppard, M. Gu, Y. Kawata, S. Kawata, "Three-dimensional transfer functions for highaperture systems," J. Opt. Soc. Am. A 11, 593-598 (1994).

183. E. Auksorius, Y. Bromberg, R. Motiejūnaitè, A. Pieretti, L. Liu, E. Coron, J. Aranda, A. M. Goldstein, B. E. Bouma, A. Kazlauskas, G. J. Tearney, "Dual-modality fluorescence and full-field optical coherence microscopy for biomedical imaging applications," Biomed. Opt. Exp. 3, 661-666 (2012).

184. J. M. Schmitt, S. L. Lee, K. M. Yung, "An optical coherence microscope with enhanced resolving power in thick tissue," Opt. Commun. 142, 203-207 (1997).

185. F. Lexer, C. K. Hitzenberger, W. Drexler, S. Molebny, H. Sattmann, M. Sticker, A. F. Fercher, "Dynamic coherent focus OCT with depth-independent transversal resolution," J. Mod. Opt. 46, 541-553 (1999).

186. B. Qi, A. P. Himmer, L. M. Gordon, X. D. Yang, L. D. Dickensheets, I. A. Vitkin, "Dynamic focus control in highspeed optical coherence tomography based on a microelectromechanical mirror," Opt. Commun. 232, 123-128 (2004).

187. A. Divetia, T.-H. Hsieh, J. Zhang, Z. Chen, M. Bachman, G.-P. Li, "Dynamically focused optical coherence tomography for endoscopic applications," Appl. Phys. Lett. 86, 103902 (2005).

188. S. Murali, J. Rolland, "Dynamic-focusing microscope objective for optical coherence tomography," International Optical Design Conf., Technical Digest (CD) (Optical Society of America), paper MD5 (2006).

189. M. Villiger, C. Pache, T. Lasser, "Dark field optical coherence microscopy," Opt. Lett. 35, 3489-3491 (2010).

190. M. E. Brezinski, Optical Coherence Tomography: Principles and Applications, Academic Press, (2006).

191. N. G. Chen, G. J. Gao, "Multi-contrast focal modulation microscopy for in vivo imaging of thick biological tissues," Opt. Exp. 20, 5 (2012).

192. N. G. Chen, C. H. Wong, C. J. R. Sheppard, "Focal modulation microscopy," Opt. Exp. 16, 1876418769 (2008). 
193. S. P. Chong, C. H. Wong, C. J. R. Sheppard, N. G. Chen, "Focal modulation microscopy: A theoretical study," Opt. Lett. 35, 1804-1806 (2010).

194. G. J. Gao, S. P. Chong, C. J. R. Sheppard, N. G. Chen, "Considerations of aperture configuration in focal modulation microscopy from the standpoint of modulation depth," J. Opt. Soc. Am. A 28, 496501 (2011).

195. C. H. Wong, S. P. Chong, C. J. R. Sheppard, N. G. Chen, "Simple spatial phase modulator for focal modulation microscopy," Appl. Opt. 48, 3237-3242 (2009).

196. S. P. Chong, C. H. Wong, K. F. Wong, C. J. R. Sheppard, N. G. Chen, "High-speed focal modulation microscopy using acousto-optical modulators," Biomed. Opt. Exp. 1, 1026-1033 (2010).

197. K. Si, W. Gong, N. G. Chen, C. J. R. Sheppard, "Enhanced background rejection in thick tissue using focal modulation microscopy with quadrant apertures," Opt. Commun. 284, 1475-1480 (2011).

198. W. Gong, K. Si, N. G. Chen, C. J. R. Sheppard, "Improved spatial resolution in fluorescence focal modulation microscopy," Opt. Lett. 34, 3508-3510 (2009).

199. W. Gong, K. Si, N. G. Chen, C. J. R. Sheppard, "Focal modulation microscopy with annular apertures: A numerical study," J. Biophoton. 3, 476-484 (2010).

200. E. Gratton, N. P. Barry, S. Beretta, A. Celli, "Multiphoton fluorescence microscopy," Methods 25, 103-110 (2001).

201. P. T. C. So, H. Kim, I. E. Kochevar, "Two-photon deep tissue ex vivo imaging of mouse dermal and subcutaneous structures," Opt. Exp. 3, 339-350 (1998).

202. Y. P. Ying, F. Liu, R. R. Alfano, "Spatial distribution of two-photon-excited fluorescence in scattering media," Appl. Opt. 38, 224-229 (1999).

203. P. Theer, M. T. Hasan, W. Denk, "Two-photon imaging to a depth of $1000 \mu \mathrm{m}$ in living brains by use of a Ti: Al2O3 regenerative amplifier," Opt. Lett. 28, 1022-1024 (2003).

204. A. Leray, J. Mertz, "Rejection of two-photon fluorescence background in thick tissue by differential aberration imaging," Opt. Exp. 14, 1056510573 (2006).

205. K. Si, W. Gong, N. G. Chen, C. J. R. Sheppard, "Two-photon focal modulation microscopy in turbid media," Appl. Phys. Lett. 99, 233702 (2011). 